IUCM94-021; CRPS-94-25

\title{
Collective modes of soliton-lattice states in double-quantum-well systems
}

\author{
R. Côté \\ Département de physique and Centre de Recherche en Physique du Solide, Université de \\ Sherbrooke, Sherbrooke, Québec, J1K 2R1, Canada. \\ L. Brey \\ Departamento Fisica Materia Condensada, Universidad Autónoma, Cantoblanco 28049 Madrid, \\ Spain \\ H. Fertig \\ Department of Physics and Astronomy,University of Kentucky,Lexington,Kentucky 40506-0055 \\ A. H. MacDonald \\ Department of Physics, Indiana University, Bloomington, Indiana 47405
}

(September 15, 2018)

\begin{abstract}
In strong perpendicular magnetic fields double-quantum-well systems can sometimes occur in unusual broken symmetry states which have interwell phase coherence in the absence of interwell hopping. When hopping is present in such systems and the magnetic field is tilted away from the normal to the quantum well planes, a related soliton-lattice state can occur which has kinks in the dependence of the relative phase between electrons in opposite layers on
\end{abstract}


the coordinate perpendicular to the in-plane component of the magnetic field. In this article we evaluate the collective modes of this soliton-lattice state in the generalized random-phase aproximation. We find that, in addition to the Goldstone modes associated with the broken translational symmetry of the soliton-lattice state, higher energy collective modes occur which are closely related to the Goldstone modes present in the spontaneously phase-coherent state. We study the evolution of these collective modes as a function of the strength of the in-plane magnetic field and comment on the possibility of using the in-plane field to generate a finite wave probe of the spontaneously phase-coherent state.

Pacs: 73.20.Dx,64.70.Rh,73.20.Mf

\section{INTRODUCTION}

Recently, there have been a number of experimental [1] and theoretical [2] investigations of the integer and fractional quantum Hall effects in double-quantum-well systems (DQWS). The additional degree of freedom due to the presence of the second well makes the phase diagram of the two-dimensional electron gas in the DQWS very rich. Technological progress

has opened the possibility of producing DQWS's of very high mobility where the spacing between the wells is small enough $(d \sim 100 \AA$ ) to be comparable with the typical electron spacing within a layer. In this regime a DQWS may differ qualitatively from a pair of isolated single-layer systems. In particular, theoretical work based on several apparently different points of view has established [3 5] that for sufficiently small separations, a novel broken symmetry can occur in DQWS's in a strong perpendicular magnetic field in which phase coherence exists between electrons in different layers even when no tunneling occurs. This broken symmetry is favored by interlayer electron-electron interactions because good interlayer correlations are guaranteed by the Pauli exclusion principle when the phase difference between the two layers is fixed. (If the phase relationship is fixed, two electrons cannot 
share the same planar coordinate even if they are in opposite layers.) The broken symmetry occurs at strong perpendicular magnetic fields because Landau level degeneracy allows the symmetry to be broken without a kinetic energy cost. The broken symmetry is expected to exist in the ground state for layer separation, $d$, smaller than a critical layer separation, $d_{c}$. Moreover, for $d<d_{c}$ the system is expected [3]6] to have a finite temperature phase transition of the Kosterlitz-Thouless type.

For realistic DQWS's with $d<d_{c}$ there is always a finite amplitude for tunneling between the two layers of the DQWS. Tunneling favors a particular phase relationship between electrons in opposite layers so that the symmetry discussed above is explicitly broken by the tunneling term in the Hamiltonian and the Kosterlitz-Thouless phase transition associated with its breaking does not occur. However, recent experiments [7 have demonstrated that a second related quantum phase transition occurs in DQWS's when the magnetic field is tilted away from the normal to the layers of the DQWS. When the component of the magnetic field which is parallel to the electron layers is finite, electrons must acquire an AharonovBohm phase when they move around paths which enclose flux from the parallel field. In one convenient gauge, the Aharonov-Bohm phase appears through phase factors in the interlayer tunneling amplitudes which depend on the planar coordinate which is perpendicular to the in-plane component of the magnetic field. The tunneling term in the Hamiltonian then favors a phase relationship between electrons in opposite layers which follows the phase of the tunneling amplitude. When the phase relationship is spatially dependent it becomes possible for electrons in opposite layers to share the same planar coordinate so that the interaction energy increases.

According to Yang et al. [8,6.9] the phase transition seen by Murphy et al. [7] results from this competition between tunneling energy and interaction energy in a parallel field. In the simplest view, the state on the small parallel field side of the transition is one where the phase relationship between the layers follows the phase of the tunneling amplitude while the state on the large parallel field side of the transition is one in which the phase is independent of the spatial coordinate and the symmetry-breaking tunneling term has 
no effect on the ground-state energy of the DQWS. A more accurate picture of this state and of the phase transition follows from the effective Hamiltonian derived by Yang et al. in which the local interaction energy cost of spatial dependence in the phase relationship between the layers is approximated by a gradient term in the energy density. Minimizing the energy in a parallel field then gives rise to a sine-Gordon equation. The state on the large parallel field side of this transition is a soliton-lattice state [8,9] which has its translational symmetry broken by the introduction of periodic kinks in the phase relationship between electrons in the two wells of the DQWS as the parallel magnetic field is increased. The effective Hamiltonian derived by Yang et al. may be mapped to thoroughly studied models (see for example Refs. [10,11]) of commensurate-incommensurate phase transitions with the parameter which tunes the transition proportional to the parallel component of the magnetic field (see below). Motivated by this mapping, we refer to the state on the weak parallel field side of the transition as the commensurate state and to the state in the absence of tunneling as the incommensurate state.

In this paper we use a generalized random-phase approximation (GRPA) to study the response functions and collective modes of the soliton-lattice state. As discussed elsewhere [6] and in further details below, the GRPA is expected to be qualitatively correct for DQWS's at total Landau level filling factor $\nu=1$. It is at this filling factor that the physical effects associated with spontaneous phase coherence are most easily observed. The use of a microscopic theory frees us from the gradient approximation and, more importantly, allows us to study higher energy excitations which may not be accurately rendered by the effective Hamiltonian. In Section II, we detail the microscopic Hamiltonian on which we base our calculations. We appeal to the strong perpendicular magnetic field in limiting the Hilbert space to the lowest Landau level in each layer and assume that the electrons are completely spin polarized. GRPA response functions are readily calculated from the HartreeFock approximation ground state of the DQWS. In Section III, we outline the HartreeFock formalism which allows us to describe both the simpler commensurate state and the more intricate soliton-lattice state. Numerical Hartree-Fock approximation results for the 
ground state are compared with expectations based on the gradient approximation effective Hamiltonian and with the theory of commensurate-incommensurate phase transitions. At strong parallel magnetic fields corrections are quantitatively important. We discuss results for the period of the soliton-lattice, the shape of individual solitons, and the ground-state energy. We show explicitly that the soliton-lattice state always has lower energy than the incommensurate phase, although the energy difference is extremely small at very strong parallel magnetic fields. We also calculate the critical strength of parallel magnetic field at which the phase transition between commensurate and soliton-lattice states occurs. In Section IV we describe the GRPA calculations. The response functions of the soliton-lattice state are complicated by its broken translational symmetry but we are able to derive formal results which are convenient for numerical calculations by taking advantage of simplifications which are due to the truncation of the Hilbert space to the lowest Landau level. The formalism is a generalization to double-layers of one developed [12] to describe the Wigner crystal state of single layer systems in strong perpendicular magnetic fields and has been applied previously [13] to double-layer systems in the case where there is no parallel field. We find that the lowest energy collective modes are Goldstone modes associated with the broken translational symmetry of the soliton-lattice state. Collective modes occur at higher energies which are closely related to the Goldstone modes of the phase-coherent broken-symmetry state in the absence of tunneling between the layers. We comment on the possibility of using a parallel magnetic field to generate a finite wave vector probe of the coherent broken symmetry state. Section V contains a brief summary of our results.

\section{MODEL HAMILTONIAN FOR DQWS'S IN A TILTED MAGNETIC FIELD}

We consider a symmetric DQWS subjected to a tilted magnetic field. We are interested in studying the ground-state properties of this system as a function of both the parallel component of the magnetic field and the separation $d$ (from center to center) between the wells at filling factor $\nu=1$. We assume throughout this paper that the perpendicular 
component of the magnetic field is kept constant. The total field is taken as $\mathbf{B}=B_{\|} \mathbf{y}+B_{\perp} \mathbf{z}$ and, in the Landau gauge, the vector potential $\mathbf{A}=\left(0, B_{\perp} x,-B_{\|} x\right)$. We consider only the case of an unbiased DQWS: the subband energies in the two layers are identical and, in the ground state, the charge is equally distributed between the two layers.

Taking the perpendicular component of the magnetic field to be strong, we restrict the Hilbert space to the first Landau level and assume the electron gas to be fully spin polarized. The many-body Hilbert space then consists of occupation number states based on a set of single-particle orbitals labeled by a layer index $i$ and a guiding center index $X$ :

$$
\phi_{i, X}(\mathbf{r})=\frac{1}{\sqrt{L_{y}}} \frac{1}{\left(\pi \ell^{2}\right)^{1 / 4}} e^{-i X y / \ell^{2}} e^{-(x-X)^{2} / 2 \ell^{2}} \chi_{i}(z),
$$

where $\ell^{2}=\hbar c / e B_{\perp}$ is the square of the magnetic length for the perpendicular component of the magnetic field and $\chi_{i}(z)$ with $i=R, L$ is the envelope wave function of the lowestenergy state centered on the right or left well. We can define a separate magnetic length associated with the parallel component of the magnetic field, $\ell_{\|}^{2}=\hbar c / e B_{\|}$. In the absence of interactions and tunneling between the wells these single particle orbitals are eigenstates of the single-particle Hamiltonian.

For the sake of limiting the number of parameters characterizing our DQWS, we make a narrow well approximation i.e. we assume that the width, $b$, of the wells is small $(b<<$ d) and treat interlayer hopping in a tight-binding [14] approximation. The single-particle problem is then characterized by the interlayer distance $d$ and a hopping integral $t$ :

$$
H_{0}=\sum_{i, X} \epsilon c_{i, X}^{\dagger} c_{i, X}-\widetilde{t} \sum_{X}\left(e^{-i X d / \ell_{\|}^{2}} c_{R, X}^{\dagger} c_{L, X}+\text { h.c. }\right) .
$$

In this approximation, the parallel component of the magnetic field is responsible for a reduction [14] in the magnitude of the hopping integral $\left(\widetilde{t}=t e^{-d^{2} \ell^{2} / 4 \ell_{\|}^{4}} \equiv t e^{-Q^{2} \ell^{2} / 4}\right)$ and for the introduction of a guiding-center-dependent phase factor, $e^{-i X d / \ell_{\|}^{2}} \equiv e^{-i Q X} . \epsilon$ is the identical subband energy of the quantum wells (including the lowest Landau-level energy $\hbar \omega_{c} / 2$, where $\left.\omega_{c}=e B_{\perp} / m c\right)$ and $Q$ is defined by

$$
Q \equiv \frac{d}{\ell_{\|}^{2}} \propto B_{\|}
$$


The parallel component of the magnetic field enters only in the non-interacting part of the Hamiltonian but, as we will see, the competition it produces with the interaction energy leads to interesting many-body physics.

\section{HARTREE-FOCK GROUND STATES IN A PARALLEL FIELD}

\section{A. Hamiltonian of the interacting 2D electron gas}

In order to evaluate the GRPA response functions we must first complete self-consistent Hartree-Fock calculations for the ground state of the DQWS. The order parameters that describe the commensurate and soliton-lattice states are defined below and we compute them using the formalism developed in Refs. [13] and [12]. Although this approach is not the simplest approach one could use in the present context, it has the advantage of being easy to implement numerically. More importantly, the results we derive here will lead to a remarkable simplification of the GRPA response function calculations.

We start be defining the operator

$$
\rho_{i j}(\mathbf{q})=\frac{1}{g} \sum_{X} e^{-i q_{x} X-i q_{x} q_{y} \ell^{2} / 2} c_{i, X}^{\dagger} c_{j, X+q_{y} \ell^{2}},
$$

where $g=S / 2 \pi \ell^{2}$ is the degeneracy of the Landau levels ( $S$ is the area of the system). The single-particle Hartree-Fock Hamiltonian can be expressed in terms of this operator. Defining $\mathbf{Q} \equiv Q \widehat{x}$ we obtain

$$
\begin{aligned}
H_{H F} & =g \epsilon \rho(0)-g \widetilde{t}\left(\rho_{R L}(\mathbf{Q})+\rho_{L R}(-\mathbf{Q})\right) \\
& +g\left(\frac{e^{2}}{\epsilon_{0} \ell}\right) \sum_{i, j} \sum_{\mathbf{q}}\left[H_{i i j j}(\mathbf{q})<\rho_{i i}(-\mathbf{q})>\rho_{j j}(\mathbf{q})-X_{i i j j}(\mathbf{q})<\rho_{i j}(-\mathbf{q})>\rho_{j i}(\mathbf{q})\right]
\end{aligned}
$$

where

$$
\begin{aligned}
& H_{i i i i}(\mathbf{q})=V_{a}(\mathbf{q})=\left(\frac{1}{q \ell}\right) e^{-q^{2} \ell^{2} / 2} \\
& H_{i i j j}(\mathbf{q})=V_{c}(\mathbf{q})=\left(\frac{1}{q \ell}\right) e^{-q^{2} \ell^{2} / 2} e^{-q d}, \\
& X_{i i i i}(\mathbf{q})=V_{b}(\mathbf{q})=\int_{0}^{\infty} d\left(q^{\prime} \ell\right) J_{0}\left(q q^{\prime} \ell^{2}\right) e^{-q^{\prime 2} \ell^{2} / 2}, \\
& X_{i i j j}(\mathbf{q})=V_{d}(\mathbf{q})=\int_{0}^{\infty} d\left(q^{\prime} \ell\right) J_{0}\left(q q^{\prime} \ell^{2}\right) e^{-q^{\prime 2} \ell^{2} / 2} e^{-q^{\prime} d},
\end{aligned}
$$


are respectively the Hartree intrawell and interwell interactions and the Fock intrawell and interwell interactions. These functions of wave vector depend only on the interlayer separation and are plotted in Ref. [13]. $J_{0}$ is a Bessel function of the first kind and $\rho(0)=\rho_{R R}(0)+\rho_{L L}(0)$ is the "density" operator. In deriving these results we have neglected processes in which electrons are scattered from one well to the other as the result of their mutual interactions. This assumption is consistent with the tight-binding approximation. In realistic systems, small terms of this type will be present but they do not add anything new to the physics.

These equations are very general and allow both positional and coherence broken symmetries. The set of quantities $<\rho_{i j}(\mathbf{q})>$ describes all possible states of the DQWS and we use them as order parameters: $<\rho_{R R}(\mathbf{q})>$ and $\left\langle\rho_{L L}(\mathbf{q})>\right.$ describe density fluctuations in each well while the interwell coherence is reflected in a non-zero value of the interwell order parameters $<\rho_{R L}(\mathbf{q})>$ and $<\rho_{L R}(\mathbf{q})>$. The states that we consider here are ones where the electron density is uniform in each well, but the interwell order parameters are allowed to vary in space along the $\hat{x}$ axis. We do not allow variation in the interwell order parameters along the $\widehat{y}$ direction. In our notation, we restrict ourselves to solutions of the Hartree-Fock equations where

$$
\begin{gathered}
<\rho_{R R}(0)>=<\rho_{L L}(0)>=\frac{1}{2}, \\
<\rho_{R L}\left(q_{x}, 0\right)>, \quad<\rho_{L R}\left(q_{x}, 0\right)>\neq 0,
\end{gathered}
$$

and all other order parameters are zero. It seems clear that the lowest-energy solution of the Hartree-Fock equations satisfies these restrictions for the situations of physical interest to us. The restriction that $<\rho_{R R}(0)>=<\rho_{L L}(0)>=1 / 2$ is enforced by the large Hartree energy cost of having unequal densities in the two layers. The only spatial dependence which we allow is that driven by the hopping term in the Hamiltonian which depends on the $\hat{x}$ coordinate. In the absence of a parallel field, we know [13] that the minimum-energy solution of the Hartree-Fock equations is spatially uniform at small layer separations but breaks translational symmetry by forming a Wigner crystal state at sufficiently large layer separations. Once the Wigner crystal state is formed, interlayer coherence is quickly lost. 
For $\nu=1$ the occurrence of a Wigner crystal state is believed to be an artifact of the Hartree-Fock approximation. In any event, our present interest is in the regime of small layer separations where we do not expect spatial dependence other than that driven by the parallel field; here we are confident that the lowest-energy Hartree-Fock state satisfies the restrictions of Eq.( (7). With these restrictions the Hartree-Fock Hamiltonian simplifies:

$$
\begin{aligned}
H_{H F} & =g\left(\epsilon-\frac{1}{2}\left(\frac{e^{2}}{\epsilon_{0} \ell}\right) V_{b}(0)\right) \rho(0)-g \widetilde{t}\left(\rho_{R L}(\mathbf{Q})+\rho_{L R}(-\mathbf{Q})\right) \\
& -g\left(\frac{e^{2}}{\epsilon_{0} \ell}\right) \sum_{q_{x}} V_{d}\left(q_{x}\right)\left[<\rho_{R L}\left(-q_{x}\right)>\rho_{L R}\left(q_{x}\right)+<\rho_{L R}\left(-q_{x}\right)>\rho_{R L}\left(q_{x}\right)\right]
\end{aligned}
$$

where $V_{b}(0)=\sqrt{\frac{\pi}{2}}$. To arrive at Eq.(8), we have added a neutralizing positive background; the energy $\epsilon$ appearing in Eq.(8) now depends on the location of this background in three dimensional space. For constant perpendicular magnetic field, the first term in the righthand-side of Eq.(8) is a constant which we absorb into the zero of energy in what follows.

\section{B. Equation of motion of the single-particle Green's function}

The Hartree-Fock Hamiltonian depends on order parameters which must be determined self-consistently. We now derive explicit self-consistent equations by considering the equation of motion of the single-particle Green's function defined by $G_{i j}\left(X, X^{\prime}, \tau\right)=-<$ $T c_{i, X}(\tau) c_{j, X^{\prime}}^{\dagger}(0)>$. Within the restricted Hartree-Fock approximation discussed above, the single-particle Green's function is diagonal in its guiding center indices and we can work 15] with a one-component Fourier transform defined by

$$
G_{i j}\left(q_{x}, \tau\right)=\frac{1}{g} \sum_{X} G_{i j}(X, X, \tau) e^{-i q_{x} X}
$$

The order parameters are related to this Green's function by $<\rho_{i j}\left(q_{x}\right)>=G_{j i}\left(q_{x}, \tau=0^{-}\right)$.

The equation of motion of the Green's function is derived using $\hbar \frac{\partial}{\partial \tau}(\cdots)=[H-\mu N, \cdots]$ where $\mu$ is the chemical potential of the electron gas and we set $\hbar=1$. We find that

$$
\begin{aligned}
& \left(i \omega_{n}+\mu\right) G_{R R}\left(q_{x}, \omega_{n}\right)+\widetilde{t} G_{L R}\left(q_{x}+Q, \omega_{n}\right)=\delta_{q_{x}, 0}-\sum_{q_{x}^{\prime}} U^{*}\left(q_{x}^{\prime}\right) G_{L R}\left(q_{x}+q_{x}^{\prime}, \omega_{n}\right) \\
& \left(i \omega_{n}+\mu\right) G_{L R}\left(q_{x}, \omega_{n}\right)+\widetilde{t} G_{R R}\left(q_{x}-Q, \omega_{n}\right)=-\sum_{q_{x}^{\prime}} U\left(q_{x}^{\prime}\right) G_{R R}\left(q_{x}-q_{x}^{\prime}, \omega_{n}\right)
\end{aligned}
$$


where the effective Hartree-Fock mean field is due to the interlayer exchange energy and is given by

$$
U\left(q_{x}\right)=\left(\frac{e^{2}}{\epsilon_{0} \ell}\right) V_{d}\left(q_{x}\right)<\rho_{R L}\left(q_{x}\right)>.
$$

Our restricted Hartree-Fock equations do not couple different guiding centers. Since we require that the charge density in each layer be uniform and independent of position, it follows that for the Hartree Fock eigenfunctions at guiding center $X$, the magnitudes of the coefficients multiplying the basis functions localized in left and right wells must be equal. The only freedom of physical relevance is the relative phase, $\theta(X)$, multiplying the basis functions. In terms of this quantity,

$$
<\rho_{R L}\left(q_{x}\right)>=\frac{1}{g} \sum_{X}<\rho(X)>e^{-i q_{x} X}=\frac{1}{g} \sum_{X}\left[\frac{1}{2} e^{i \theta(X)}\right] e^{-i q_{x} X}
$$

where $\left\langle\rho(X)>=<c_{R, X}^{\dagger} c_{L, X}>\right.$ is the interwell order parameter in $X$ space.

At this point, it is more convenient [8,9] to define a new phase $\widetilde{\theta}(X)$ by

$$
\widetilde{\theta}(X)=\theta(X)-Q X
$$

In the commensurate state, $\widetilde{\theta}(X) \equiv 0$, while in the soliton-lattice state it is a periodic function of $X$. It is advantageous to make use of this periodicity and redefine the interwell order parameter as

$$
<\widetilde{\rho}_{R L}\left(q_{x}\right)>\equiv<\rho_{R L}\left(q_{x}+Q\right)>=\frac{1}{2 g} \sum_{X} e^{-i q_{x} X} e^{i \widetilde{\theta}(X)}
$$

which is the Fourier transform of $<\widetilde{\rho}(X)>=<\rho(X)>e^{-i Q X}=\frac{1}{2} e^{\tilde{i} \widetilde{\theta}(X)}$.

In our notation the commensurate-incommensurate phase transition of Ref. [8] is described in the following way. The commensurate phase (C phase) at weak parallel magnetic fields has $\theta(X)=Q X$; the phase of the wavefunction follows the phase of the hopping matrix element just as it would for non-interacting electrons. For this state $\tilde{\theta}(X)=0$ and so only $<\widetilde{\rho}_{R L}(0)>=\frac{1}{2}$ is different from zero. The commensurate phase has an energy per particle 


$$
E_{C}=-\tilde{t}-\frac{1}{4}\left(\frac{e^{2}}{\epsilon_{0} \ell}\right) V_{d}(Q),
$$

which increases monotonically with magnetic field. In the opposite limit of strong parallel magnetic fields, the phase does not follow the imposed periodicity and we expect $\theta(X)=0$. Thus, $\widetilde{\theta}(X)=-Q X$ or, equivalently, only $<\widetilde{\rho}_{R L}(-\mathbf{Q})>=\frac{1}{2}$ is non-zero. The energy per particle in the incommensurate phase (I phase) is

$$
E_{I}=-\frac{1}{4}\left(\frac{e^{2}}{\epsilon_{0} \ell}\right) V_{d}(0),
$$

and is clearly independent of the parallel magnetic field. There is a critical parallel magnetic

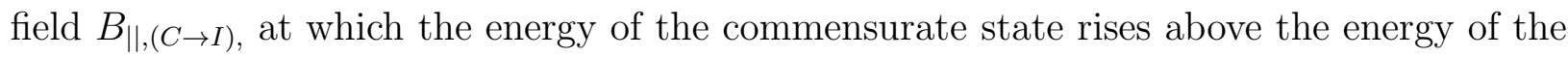
incommensurate state. As pointed out in Ref. [8], however, before this critical magnetic field is reached it becomes energetically favorable to introduce kinks (solitons) where $\widetilde{\theta}(X)$ changes by $2 \pi$; in the ground state the solitons are arranged periodically. We refer to this state as the soliton-lattice state (S phase). For very strong parallel magnetic fields the solitons are smooth and spaced by $2 \pi / Q$ so that $\tilde{\theta}(X) \approx-Q X$ and the soliton-lattice state asymptotically approaches the incommensurate state. In Ref. [8] this phase transition is described using a gradient approximation for the exchange energy which allows the ground-state problem to be mapped to well-studied models of commensurate-incommensurate phase transitions 10,11. In the Appendix, we briefly review the most relevant results from these models and discuss the relationship between the gradient-approximation theory for the ground state and the Hartree-Fock approximation. At the critical parallel field for the transition between commensurate and soliton-lattice states, $B_{\|,(C \rightarrow S)}$, the system admits a single soliton and $\widetilde{\theta}(X)$ is a solution of the sine-Gordon equation. (See the appendix for further details.) As the parallel magnetic field increases above this transition, the solitons proliferate and it is necessary to take their interactions into account. This is best done numerically [10,11] even in the gradient approximation. Below we describe our numerical Hartree-Fock calculations for the soliton-lattice ground state.

In the ground state of the soliton-lattice phase, we have the periodicity $e^{\tilde{i} \widetilde{\theta}\left(X+L_{S}\right)}=e^{i \widetilde{\theta}(X)}$ 
where $L_{S}$, the period of the soliton-lattice, is chosen to minimize the energy. It follows that if we define a soliton wave vector by

$$
Q_{S}=\frac{2 \pi}{L_{S}}
$$

the only non-zero order parameters are $<\widetilde{\rho}_{R L}\left(n Q_{S}\right)>, n=0, \pm 1, \pm 2, \ldots$ (We can choose the origin for the $\hat{x}$ coordinate so that these order parameters are real.) We can simplify the notation of Eqs.(10) by redefining the interwell single-particle Green's function as

$$
\widetilde{G}_{L R}\left(q_{x}, \omega_{n}\right) \equiv G_{L R}\left(q_{x}+Q, \omega_{n}\right) .
$$

Defining $\widetilde{G}_{L R}\left(n, \omega_{n}\right) \equiv \widetilde{G}_{L R}\left(n Q_{S}, \omega_{n}\right), G_{R R}\left(n, \omega_{n}\right) \equiv G_{R R}\left(n Q_{S}, \omega_{n}\right), \widehat{u}(n) \equiv \delta_{n, 0}$ and the real matrix

$$
F(n, m) \equiv \widetilde{t} \delta_{n, m}+\left(\frac{e^{2}}{\epsilon_{0} \ell}\right) V_{d}\left((n-m) Q_{S}+Q\right)<\widetilde{\rho}_{R L}(n-m)>
$$

we have, in an obvious matrix notation,

$$
\begin{aligned}
\left(i \omega_{n}+\mu\right) G_{R R}\left(\omega_{n}\right)+F^{T} \widetilde{G}_{L R}\left(\omega_{n}\right) & =\widehat{u}, \\
\left(i \omega_{n}+\mu\right) \widetilde{G}_{L R}\left(\omega_{n}\right)+F G_{R R}\left(\omega_{n}\right) & =0 .
\end{aligned}
$$

These equations are readily decoupled:

$$
\begin{aligned}
& {\left[\left(i \omega_{n}+\mu\right)^{2} I-B\right] G_{R R}\left(\omega_{n}\right)=\left(i \omega_{n}+\mu\right) \widehat{u},} \\
& {\left[\left(i \omega_{n}+\mu\right)^{2} I-B\right] \widetilde{G}_{L R}\left(\omega_{n}\right)=-F \widehat{u},}
\end{aligned}
$$

where the real and symmetric matrix $B$ is defined by

$$
B=F^{T} F=F F^{T}
$$

$B$ can be diagonalized by an orthogonal transformation: $B=U \Omega U^{T}$ where $\Omega$ is the diagonal matrix of the eigenvalues of $B$ with elements $\Omega_{i}$. Solving for the order parameters, we find at finite temperature

$$
\left\langle\rho_{R R}(n)\right\rangle=\frac{1}{2} \sum_{i} U_{n, i} U_{i, 0}^{T}\left[f\left(E_{i,-}\right)+f\left(E_{i,+}\right)\right]
$$


and

$$
\left\langle\widetilde{\rho}_{R L}(n)\right\rangle=\frac{1}{2} \sum_{i, k} \frac{U_{n, i} U_{i, k}^{T} F(k, 0)}{\left|E_{i}\right|}\left[f\left(E_{i,-}\right)-f\left(E_{i,+}\right)\right]
$$

where $f(x)=\frac{1}{e^{\beta(x-\mu)}+1}$ is the Fermi function and

$$
E_{i, \pm}= \pm \sqrt{\Omega_{i}}
$$

In order to avoid density fluctuations at $T=0 K$, i.e. in order to have $\left\langle\rho_{i i}(0)\right\rangle=$ $\frac{1}{2},\left\langle\rho_{i i}(n \neq 0)\right\rangle=0(i=R, L)$, we must have $\mu(T=0)=0$ so that the Fermi factors equal one for the negative energy solutions and zero for the positive energy solutions. Then $\left\langle\rho_{i i}(n)\right\rangle=\frac{1}{2} \sum_{j} U_{n, j} U_{j, 0}^{\dagger}=\frac{1}{2} \delta_{n, 0}$ and, in matrix notation,

$$
\left\langle\widetilde{\rho}_{R L}\right\rangle=\frac{1}{2} U \Omega^{-\frac{1}{2}} U^{T} F \widehat{\mathbf{u}},
$$

where $\Omega^{1 / 2}$ represents the diagonal matrix of $\Omega_{i}^{1 / 2}$ values. From this last equation, we can directly show that the interwell order parameter obeys the sum rule

$$
\begin{aligned}
\sum_{n}\left|\left\langle\widetilde{\rho}_{R L}(n)\right\rangle\right|^{2} & =\left\langle\widetilde{\rho}_{R L}\right\rangle^{T}\left\langle\widetilde{\rho}_{R L}\right\rangle \\
& =\left(\frac{1}{2} \widehat{\mathbf{u}}^{T} F^{T} U \Omega^{-\frac{1}{2}} U^{T}\right)\left(\frac{1}{2} U \Omega^{-\frac{1}{2}} U^{T} F \widehat{\mathbf{u}}\right) \\
& =\frac{1}{4} \widehat{\mathbf{u}}^{T} F^{T} B^{-1} F \widehat{\mathbf{u}} \\
& =\frac{1}{4}
\end{aligned}
$$

Eqs.(22),(25), (26) represent a set of self-consistent, coupled equations that we must solve in an iterative way until a stable solution is found for a given value of $Q_{S}, Q$ and $t$. The calculation must then be repeated for different values of $Q_{S}$ until we find the solution which minimizes the HF ground-state energy per particle

$$
E=-2 \widetilde{t}<\widetilde{\rho}_{R L}(0)>-\left(\frac{e^{2}}{\epsilon_{0} \ell}\right) \sum_{n} V_{d}\left(n Q_{S}+Q\right)\left\langle\widetilde{\rho}_{R L}(n)\right\rangle^{2}
$$

Given the order parameters, we can easily find $\widetilde{\theta}(X)$ from the equation

$$
<\widetilde{\rho}_{R L}(X)>=\frac{1}{2} e^{i \widetilde{\theta}(X)}=\sum_{n} e^{i n Q_{S} X}<\widetilde{\rho}_{R L}(n)>.
$$

The Hartree-Fock eigenvalues $E_{ \pm}(X)$ (see appendix) are related to the order parameters by 


$$
E_{ \pm}(X)= \pm\left|\widetilde{t}+\sum_{n} V_{d}\left(n Q_{S}+Q\right)<\widetilde{\rho}_{R L}(n)>e^{i n Q_{S} X}\right|
$$

Commensurate and incommensurate states also represent extrema of the Hartree-Fock energy functional and therefore are self-consistent solutions of the Hartree-Fock equations. For commensurate and incommensurate states respectively we find that

$$
E_{C}^{ \pm}(X)= \pm\left(\widetilde{t}+\frac{1}{2} V_{d}(Q)\right)
$$

and

$$
E_{I}^{ \pm}(X)= \pm\left|\widetilde{t}+\frac{1}{2} V_{d}(0) e^{-i Q X}\right|
$$

\section{Numerical results for the Hartree-Fock Approximation Ground State}

In Fig. 1, we plot the energies of the $\mathrm{C}, \mathrm{I}$ and $\mathrm{S}$ phases for $(\mathrm{a}) t /\left(e^{2} / \ell\right)=0.01, d / \ell=1.0$ and $(\mathrm{b}) t /\left(e^{2} / \ell\right)=0.005, d / \ell=1.877$. The dotted line represents the gradient approximation for the commensurate state energy

$$
E_{C,(\text { grad })} \simeq-\widetilde{t}-\frac{1}{4}\left(\frac{e^{2}}{\epsilon_{0} \ell}\right) V_{d}(0)+\pi \ell^{2} \rho_{S} Q^{2} .
$$

The stiffness $\rho_{S}$ is easily obtained from $V_{d}(Q)$ using Eq.(A14). We find for (a) $\pi \rho_{S}=$ $0.0195\left(e^{2} / \ell\right)$ and $(\mathrm{b}) \pi \rho_{S}=0.00751\left(e^{2} / \ell\right)$. The gradient approximation is very close to the HF energy for $E_{C}$. Analytic results from the gradient approximation for the the values of $Q$ at which the commensurate and incommensurate states are equal in energy $\left(Q_{C \rightarrow I}\right)$ and at which the soliton state first becomes stable $\left(Q_{C \rightarrow S}\right)$ are also in good agreement with the the HF values. Numerically, we find that for (a) $Q_{C \rightarrow S} / Q_{C \rightarrow I}=0.91$, and for (b) $Q_{C \rightarrow S} / Q_{C \rightarrow I}=$ 0.95 while in the gradient approximation, this ratio is $Q_{C \rightarrow S} / Q_{C \rightarrow I}=2 \sqrt{2} / \pi=0.90$.

We also see in Fig. 1 that the energy of the soliton phase is always lower than the energy of the incommensurate phase so that this phase is never thermodynamically stable in the Hartree-Fock approximation. Of course, as $Q$ becomes very large, the order parameters in the soliton phase become asymptotically close to those of the $I$ phase and the energies of the 
two phases becomes nearly identical. One should also notice that the difference in energy between the soliton and incommensurate or commensurate phase is of the order of $1 \%$ at most!

The behavior of $\tilde{\theta}(X)$ over one period of the soliton lattice is shown in Fig. 2. As expected, the width of the soliton is very large right at the transition and then decreases as the parallel magnetic field increases. In the large $Q$ limit, $\widetilde{\theta}(X) \rightarrow-Q X+\pi$, which is the incommensurate solution (the constant $\pi$ here comes from the fact that we have made an overall phase convention which forces the soliton solution to vary from $2 \pi$ to 0 over the interval $\left.\left(-L_{S} / 2, L_{S} / 2\right)\right)$.

The dotted line in (a) and (b) represents the sine-Gordon solution $\tilde{\theta}(X)=$ $4 \tan ^{-1}\left[e^{-X / \xi}\right]$, where $\xi=\sqrt{2 \pi \rho_{S} \ell^{2} / \tilde{t}}$ (see Eq.(A19)). Fig. 3, is a plot of the soliton wave vector $Q_{S} \equiv 2 \pi / L_{S}$ as a function of $Q \ell$. As the parallel magnetic field increases, the soliton wave vector asymptotically approaches $Q$ as required to approach the incommensurate state solution.

Fig. 4 shows the shape of the upper band $E_{+}(X)$ over one period $L_{S}$ for two extreme values of $Q \ell$. The dispersion of the soliton band structure seen in (a) weakens as the parallel magnetic field is increased (see (b)). In the Hartree-Fock approximation the charge gap at $\nu=1$, the minimum energy to make a particle-hole pair, is the sum of the maximum and the minimum values of these bands. (Remember that the Hartree-Fock eigenvalues at $\nu=1$ occur in opposite energy pairs.) It is the charge gap which is measured in the transport [7] experiments. We see in Fig. 4 that, within the Hartree-Fock approximation, the effect of interactions on the charge gap is enormous. The hopping energy is here of order $0.01\left(e^{2} / \ell\right)$ while the band energy is one order of magnitude higher! However, we find that the HartreeFock charge gap is only weakly dependent on the parallel component of the magnetic field, in complete disagreement with experiment. This behavior is actually expected. The lowestenergy charged excitations are believed [8,6,9, [16] to be spin-textures and not the simple single-particle excitations whose energies are obtained from the Hartree-Fock calculations. The spin textures in double-layer systems are analogous to the skyrmion spin-textures which 
are known to be the lowest energy charged excitations [17, 18 of a single-layer system which is not spin-polarized.

Fig. 5, shows the commensurate energy for various values of the bare hopping $t$. If we approximate the critical wave vector for the commensurate-soliton transition by that for the $C \rightarrow I$ transition (this is not a bad approximation as we saw above), we find the curve given in Fig. 6. Notice that because of the renormalization of $t$ to $\tilde{t}$, there is always a critical value of $Q$ at which this transition occurs. The angle that the magnetic field makes with the DQWS at the transition is given by $\tan \left(\theta_{c}\right)=Q \ell /(d / \ell)$. In Fig. 6, we plot the critical angle for the transition at $d / \ell=1$. It is clear that the critical angle has a square-root dependence on $t$ at small $t$. The same result was [8] obtained in the gradient approximation. Experimentally the critical tilt angle is identified with a feature in the dependence of the charge gap on tilt angle and it appears [7] that the tilt angle has a linear dependence on $t$. Moreover, for given $t$ the critical angle appears to be overestimated by both the HartreeFock approximation and by the gradient approximation when the stiffness coefficient, $\rho_{S}$, (see Eq.(A14)) is calculated in the Hartree-Fock approximation. [8] For example, the critical angle that would be given for the parameters $d=1.877, t=0.005$ (see Fig.1 (b) above) which are similar to those for the sample studied in Ref. [7], is approximately of $21^{\circ}$. This value is about 2.6 times greater than that of the experiment $\left(8^{\circ}\right)$. As discussed elsewhere 99 we believe that this quantitative discrepancy is due to quantum fluctuations not included in the Hartree-Fock approximation. For larger values of $t$ these fluctuations are suppressed and we expect, in agreement with experiment, that critical tilt angles will agree more closely with the Hartree-Fock approximation.

\section{RESPONSE FUNCTIONS OF THE COMMENSURATE AND SOLITON PHASES IN THE GRPA}

We now consider the calculation of the response functions of the DQWS in the presence of the tilted magnetic field. The total response is characterized in general by sixteen coupled 
susceptibilities:

$$
\chi_{i j k l}\left(\mathbf{q}, \mathbf{q}^{\prime}, \tau\right)=-g<T \widehat{\rho}_{i j}(\mathbf{q}, \tau) \widehat{\rho}_{k l}\left(-\mathbf{q}^{\prime}, 0\right)>
$$

where $\hat{\rho}_{i j}=\rho-<\rho_{i j}>$. It turns out (as in the ground-state calculation), that the equations of motion for the susceptibilities have a simpler form if we work with the shifted operators $\widetilde{\rho}_{R L}, \widetilde{\rho}_{L R}$ instead of the unshifted ones. We define the $4 \times 4$ matrix

$\Gamma\left(\mathbf{q}, \mathbf{q}^{\prime}, \tau\right) \equiv$

$$
\left(\begin{array}{cccc}
\chi_{R R R R}\left(\mathbf{q}, \mathbf{q}^{\prime}, \tau\right) & \chi_{R R L R}\left(\mathbf{q}, \mathbf{q}^{\prime}+\mathbf{Q}, \tau\right) & \chi_{R R R L}\left(\mathbf{q}, \mathbf{q}^{\prime}-\mathbf{Q}, \tau\right) & \chi_{R R L L}\left(\mathbf{q}, \mathbf{q}^{\prime}, \tau\right) \\
\chi_{R L R R}\left(\mathbf{q}+\mathbf{Q}, \mathbf{q}^{\prime}, \tau\right) & \chi_{R L L R}\left(\mathbf{q}+\mathbf{Q}, \mathbf{q}^{\prime}+\mathbf{Q}, \tau\right) & \chi_{R L R L}\left(\mathbf{q}+\mathbf{Q}, \mathbf{q}^{\prime}-\mathbf{Q}, \tau\right) & \chi_{R L L L}\left(\mathbf{q}+\mathbf{Q}, \mathbf{q}^{\prime}, \tau\right) \\
\chi_{L R R R}\left(\mathbf{q}-\mathbf{Q}, \mathbf{q}^{\prime}, \tau\right) & \chi_{L R L R}\left(\mathbf{q}-\mathbf{Q}, \mathbf{q}^{\prime}+\mathbf{Q}, \tau\right) & \chi_{L R R L}\left(\mathbf{q}-\mathbf{Q}, \mathbf{q}^{\prime}-\mathbf{Q}, \tau\right) & \chi_{L R L L}\left(\mathbf{q}-\mathbf{Q}, \mathbf{q}^{\prime}, \tau\right) \\
\chi_{L L R R}\left(\mathbf{q}, \mathbf{q}^{\prime}, \tau\right) & \chi_{L L L R}\left(\mathbf{q}, \mathbf{q}^{\prime}+\mathbf{Q}, \tau\right) & \chi_{L L R L}\left(\mathbf{q}, \mathbf{q}^{\prime}-\mathbf{Q}, \tau\right) & \chi_{L L L L}\left(\mathbf{q}, \mathbf{q}^{\prime}, \tau\right)
\end{array}\right)
$$

where again $\mathbf{Q}=Q \widehat{\mathbf{x}}$.

The generalized random-phase approximation (GRPA) for the 2DEG in a strong magnetic field with broken translational symmetry is discussed in detail in Ref. [12]. The application of this formalism to the present problem presents no major difficulty although the algebra is knotty. Below we briefly sketch the derivation of the expressions we use for the numerical evaluation of the GRPA response functions.

We first define the functions

$$
\begin{aligned}
f\left(q_{x}\right) & =\tilde{t} \delta_{q_{x}, 0}+\left(\frac{e^{2}}{\varepsilon_{0} \ell}\right) V_{d}\left(q_{x}+Q\right)<\widetilde{\rho}_{R L}\left(q_{x}\right)>, \\
F\left(\mathbf{q}-\mathbf{q}^{\prime}\right) & =f\left(q_{x}-q_{x}^{\prime}\right) e^{-i\left(q_{x}-q_{x}^{\prime}+Q\right) q_{y} \ell^{2} / 2} \delta_{q_{y}, q_{y}^{\prime}}, \\
F^{T}\left(\mathbf{q}-\mathbf{q}^{\prime}\right) & =F\left(\mathbf{q}^{\prime}-\mathbf{q}\right), \\
K\left(\mathbf{q}-\mathbf{q}^{\prime}\right) & =<\widetilde{\rho}_{R L}\left(q_{x}-q_{x}^{\prime}\right)>e^{-i\left(q_{x}-q_{x}^{\prime}+Q\right) q_{y} \ell^{2} / 2} \delta_{q_{y}, q_{y}^{\prime}}, \\
K^{T}\left(\mathbf{q}-\mathbf{q}^{\prime}\right) & =K\left(\mathbf{q}^{\prime}-\mathbf{q}\right),
\end{aligned}
$$

and the $4 \times 4$ matrices 


$$
\begin{aligned}
P\left(\mathbf{q}, \mathbf{q}^{\prime}\right) & =\left[\begin{array}{cccc}
0 & -F^{T}\left(\mathbf{q}-\mathbf{q}^{\prime}\right) & F\left(\mathbf{q}-\mathbf{q}^{\prime}\right) & 0 \\
-F^{*}\left(\mathbf{q}-\mathbf{q}^{\prime}\right) & 0 & 0 & F\left(\mathbf{q}-\mathbf{q}^{\prime}\right) \\
F^{\dagger}\left(\mathbf{q}-\mathbf{q}^{\prime}\right) & 0 & 0 & -F^{T}\left(\mathbf{q}-\mathbf{q}^{\prime}\right) \\
0 & F^{\dagger}\left(\mathbf{q}-\mathbf{q}^{\prime}\right) & -F^{*}\left(\mathbf{q}-\mathbf{q}^{\prime}\right) & 0
\end{array}\right], \\
S\left(\mathbf{q}, \mathbf{q}^{\prime}\right) & =\left[\begin{array}{cccc}
0 & -K^{T}\left(\mathbf{q}-\mathbf{q}^{\prime}\right) & K\left(\mathbf{q}-\mathbf{q}^{\prime}\right) & 0 \\
-K^{*}\left(\mathbf{q}-\mathbf{q}^{\prime}\right) & 0 & 0 & K\left(\mathbf{q}-\mathbf{q}^{\prime}\right) \\
K^{\dagger}\left(\mathbf{q}-\mathbf{q}^{\prime}\right) & 0 & 0 & -K^{T}\left(\mathbf{q}-\mathbf{q}^{\prime}\right) \\
0 & K^{\dagger}\left(\mathbf{q}-\mathbf{q}^{\prime}\right) & -K^{*}\left(\mathbf{q}-\mathbf{q}^{\prime}\right) & 0
\end{array}\right], \\
X\left(\mathbf{q}, \mathbf{q}^{\prime}\right) & {\left[\begin{array}{cccc}
V_{b}(\mathbf{q}) \delta_{\mathbf{q}, \mathbf{q}^{\prime}} \\
0 & V_{d}(\mathbf{q}+\mathbf{Q}) \delta_{\mathbf{q}, \mathbf{q}^{\prime}} & 0 & 0 \\
0 & 0 & V_{d}(\mathbf{q}-\mathbf{Q}) \delta_{\mathbf{q}, \mathbf{q}^{\prime}} & 0 \\
0 & 0 & 0 & V_{b}(\mathbf{q}) \delta_{\mathbf{q}, \mathbf{q}^{\prime}}
\end{array}\right], }
\end{aligned}
$$

and

$$
H\left(\mathbf{q}, \mathbf{q}^{\prime}\right)=\left[\begin{array}{cccc}
V_{a}(\mathbf{q}) \delta_{\mathbf{q}, \mathbf{q}^{\prime}} & 0 & 0 & V_{c}(\mathbf{q}) \delta_{\mathbf{q}, \mathbf{q}^{\prime}} \\
0 & 0 & 0 & 0 \\
0 & 0 & 0 & 0 \\
V_{c}(\mathbf{q}) \delta_{\mathbf{q}, \mathbf{q}^{\prime}} & 0 & 0 & V_{a}(\mathbf{q}) \delta_{\mathbf{q}, \mathbf{q}^{\prime}}
\end{array}\right] .
$$

Using $\hbar \frac{\partial}{\partial \tau}(\cdots)=[H-\mu N, \cdots]$ where $H$ is the Hartree-Fock Hamiltonian (Eq.(8)), we find that the bare susceptibility $\Gamma^{0}$ (corresponding to a one-loop approximation with the propagators evaluated in the HFA) satisfies the matrix equation

$$
\sum_{\mathbf{q}^{\prime \prime}}\left[I(\omega+i \delta) \delta_{\mathbf{q}, \mathbf{q}^{\prime \prime}}-P\left(\mathbf{q}, \mathbf{q}^{\prime \prime}\right)\right] \Gamma^{0}\left(\mathbf{q}^{\prime \prime}, \mathbf{q}^{\prime}, \omega\right)=S\left(\mathbf{q}, \mathbf{q}^{\prime}\right) .
$$

Here $I$ is a unit matrix. We note that this bare susceptibility is independent of the value of $q_{y}$ since the propagators do not involve $q_{y}$. This is no longer the case when we add bubbles and ladder diagrams. The summation of the ladder diagrams is given by the equation $(\widehat{\Gamma}$ is the irreducible polarization) 


$$
\widehat{\Gamma}\left(\mathbf{q}, \mathbf{q}^{\prime}, \omega\right)=\Gamma^{0}\left(\mathbf{q}, \mathbf{q}^{\prime}, \omega\right)-\sum_{\mathbf{q}^{\prime \prime}, \mathbf{q}^{\prime \prime \prime}} \Gamma^{0}\left(\mathbf{q}, \mathbf{q}^{\prime \prime}, \omega\right) X\left(\mathbf{q}^{\prime \prime}, \mathbf{q}^{\prime \prime \prime}\right) \widehat{\Gamma}\left(\mathbf{q}^{\prime \prime \prime}, \mathbf{q}^{\prime}, \omega\right)
$$

so that the full GRPA susceptibility is a sum of bubbles diagrams containing the irreducible polarization and is given by

$$
\Gamma\left(\mathbf{q}, \mathbf{q}^{\prime}, \omega\right)=\widehat{\Gamma}\left(\mathbf{q}, \mathbf{q}^{\prime}, \omega\right)+\sum_{\mathbf{q}^{\prime \prime}, \mathbf{q}^{\prime \prime \prime}} \widehat{\Gamma}\left(\mathbf{q}, \mathbf{q}^{\prime \prime}, \omega\right) H\left(\mathbf{q}^{\prime \prime}, \mathbf{q}^{\prime \prime \prime}\right) \Gamma\left(\mathbf{q}^{\prime \prime \prime}, \mathbf{q}^{\prime}, \omega\right)
$$

The $q_{y}$ dependence enters only through the interaction $H$ and $X$ and so it is obvious that $\Gamma\left(\mathbf{q}, \mathbf{q}^{\prime}, \omega\right)=\Gamma\left(q_{x}, q_{x}^{\prime} ; q_{y}, \omega\right) \delta_{q_{y}, q_{y}}$.

Because of the periodicity of the system, we must have $\Gamma\left(\mathbf{q}, \mathbf{q}^{\prime}, \omega\right)=\Gamma(\mathbf{k}+n \mathbf{Q}, \mathbf{k}+m \mathbf{Q}, \omega)$, where $n, m=0, \pm 1, \pm 2, \ldots$ and $\mathbf{k}$ is a vector in the first Brillouin zone of the modulated structure i.e. $k_{x} \in\left[\frac{-\pi}{L_{s}}, \frac{\pi}{L_{s}}\right]$ and $k_{y}$ is unrestricted (the Brillouin zone is effectively onedimensional). This implies that all elements in the above matrices are themselves matrices with respect to the indices $n, m$. Eqs. (41)-(43) can then be written in a purely matrix form and the structure of the GRPA becomes much more transparent. One must however be very careful not to interchange the order of these matrices since they do not commute! Combining these three equations, we arrive at

$$
[I(\omega+i \delta)-R] \Gamma=S
$$

where

$$
R=P-S(X-H)
$$

or, more explicitly

$$
R=\left[\begin{array}{cccc}
0 & -F^{T}+K^{T} X_{22} & F-K X_{33} & 0 \\
-F^{*}+K^{*} X_{11}-K^{*} H_{11}+K H_{14} & 0 & 0 & -R_{21}^{*} \\
F^{\dagger}-K^{\dagger} X_{11}+K^{\dagger} H_{11}-K^{T} H_{14} & 0 & 0 & -R_{31}^{*} \\
0 & -R_{12}^{*} & -R_{13}^{*} & 0
\end{array}\right] .
$$

Looking at the form of the matrix $R$, it is quite clear that Eq.(44) can be further simplified. After some algebra, we find that 


$$
\left[I(\omega+i \delta)^{2}-\Lambda\right] \Gamma=\Xi
$$

where

$$
\Lambda=\left[\begin{array}{cccc}
\Lambda_{11} & 0 & 0 & \Lambda_{14} \\
0 & \Lambda_{22} & \Lambda_{23} & 0 \\
0 & \Lambda_{32} & \Lambda_{33} & 0 \\
\Lambda_{14}^{*} & 0 & 0 & \Lambda_{11}^{*}
\end{array}\right]
$$

with

$$
\begin{aligned}
& \Lambda_{11}=R_{12} R_{21}+R_{13} R_{31}, \\
& \Lambda_{22}=R_{21} R_{12}+R_{21}^{*} R_{12}^{*}, \\
& \Lambda_{33}=R_{31} R_{13}+R_{31}^{*} R_{13}^{*}, \\
& \Lambda_{14}=-R_{12} R_{21}^{*}-R_{13} R_{31}^{*}, \\
& \Lambda_{23}=R_{21} R_{13}+R_{21}^{*} R_{13}^{*}, \\
& \Lambda_{32}=R_{31} R_{12}+R_{31}^{*} R_{12}^{*},
\end{aligned}
$$

and

$$
\Xi=\left[\begin{array}{cccc}
R_{12} K^{*}-R_{13} K^{\dagger} & -(\omega+i \delta) K^{T} & (\omega+i \delta) K & -R_{12} K+R_{13} K^{T} \\
-(\omega+i \delta) K^{*} & R_{21} K^{T}+R_{21}^{*} K^{\dagger} & -R_{21} K-R_{21}^{*} K^{*} & (\omega+i \delta) K \\
(\omega+i \delta) K^{\dagger} & R_{31} K^{T}+R_{31}^{*} K^{\dagger} & -R_{31} K-R_{31}^{*} K^{*} & -(\omega+i \delta) K^{T} \\
-R_{12}^{*} K^{*}+R_{13}^{*} K^{\dagger} & (\omega+i \delta) K^{\dagger} & -(\omega+i \delta) K^{*} & R_{12}^{*} K-R_{13}^{*} K^{T}
\end{array}\right]
$$

In order to make connection with the pseudospin language used in Ref. [8], we define the density and pseudospin operators by (remember that real spins are assumed fully polarized)

$$
\begin{gathered}
n \equiv \rho_{R R}+\rho_{L L}, \\
S_{Z} \equiv \frac{1}{2}\left(\rho_{R R}-\rho_{L L}\right) .
\end{gathered}
$$

For example, we can now construct the density response function as well as the longitudinal and transverse pseudospin response functions

$$
\begin{aligned}
\chi_{n n} & =\chi_{R R R R}+\chi_{R R L L}+\chi_{L L R R}+\chi_{L L L L}, \\
\chi_{S_{Z} S_{Z}} & =\frac{1}{4}\left(\chi_{R R R R}-\chi_{R R L L}-\chi_{L L R R}+\chi_{L L L L}\right), \\
\chi_{+-} & =\chi_{R L L R} .
\end{aligned}
$$


For the functions of Eq.(52), we need to solve for

$$
\chi_{L}=\left[\begin{array}{cc}
\chi_{R R R R} & \chi_{R R L L} \\
\chi_{L L R R} & \chi_{L L L L}
\end{array}\right],
$$

and

$$
\chi_{T}=\left[\begin{array}{cc}
\chi_{R L L R} & \chi_{R L R L} \\
\chi_{L R L R} & \chi_{L R R L}
\end{array}\right] .
$$

With the definitions

$$
\begin{gathered}
R_{A}=\left[\begin{array}{cc}
R_{12} & R_{13} \\
-R_{12}^{*} & -R_{13}^{*}
\end{array}\right], \\
R_{B}=\left[\begin{array}{cc}
R_{21} & -R_{21}^{*} \\
R_{31} & -R_{31}^{*}
\end{array}\right],
\end{gathered}
$$

and

$$
K_{A}=\left[\begin{array}{cc}
K^{*} & -K \\
-K^{\dagger} & K^{T}
\end{array}\right],
$$

we arrive at the form of the equations which we have solved numerically:

$$
\begin{aligned}
& {\left[I(\omega+i \delta)^{2}-R_{A} R_{B}\right] \chi_{L}=R_{A} K_{A}} \\
& {\left[I(\omega+i \delta)^{2}-R_{B} R_{A}\right] \chi_{T}=R_{B} K_{A}^{\dagger}}
\end{aligned}
$$

Notice that $R_{12}$ is not simply related to $R_{21}$ so that there is no simple relationship between $R_{A}$ and $R_{B}$. These two matrices do not commute in the general case. We stress once again that $R_{A}$ and $R_{B}$ are not simple $2 \times 2$ matrices since each of their elements is itself an infinite matrix. Inverting Eq. (58), we easily arrive at

$$
\begin{aligned}
& \chi_{L}=R_{A}\left[I(\omega+i \delta)^{2}-R_{B} R_{A}\right]^{-1} K_{A}, \\
& \chi_{T}=\left[I(\omega+i \delta)^{2}-R_{B} R_{A}\right]^{-1} R_{B} K_{A}^{\dagger} .
\end{aligned}
$$

Where now the order of the matrices $R_{A}$ and $R_{B}$ are the same in both equations and the matrix $R_{B} R_{A}$ is real. This shows clearly that all response functions do share the same poles 
although each pole will in general have different residues for different response functions. The collective modes associated with the longitudinal response functions are thus the same as those associated with the transverse response functions. We can of course solve, in the same way, for all other response functions in Eq.(35).

If we diagonalize the matrix $R_{B} R_{A}$ by

$$
\left(R_{B} R_{A}\right) U=U \Omega
$$

where $\Omega$ is a diagonal matrix of the eigenvalues and $U$ the matrix of eigenvectors, we can write

$$
\left(\chi_{T}\right)_{i j}=\sum_{l, m, k} \frac{U_{i l}\left(U^{-1}\right)_{l m}\left(R_{B}\right)_{m k}\left(K_{A}^{\dagger}\right)_{k j}}{(\omega+i \delta)^{2}-\Omega_{l}} .
$$

When $q_{y}=0$, all matrices are real and the above equations simplify considerably. In this case, we can easily show that

$$
\begin{gathered}
\chi_{n n}=0, \\
\chi_{S_{z} S_{z}}=\chi_{R R R R} .
\end{gathered}
$$

There is thus no density response to potentials which are independent of the coordinate along the parallel magnetic field.

The numerical procedure to compute the response functions and find the collective excitations is very simple. Once the order parameters are known from the HFA described in the previous section, we can compute the matrices $R_{B}, R_{A}$, and $K_{A}$. We then diagonalize the real matrix $R_{B} R_{A}$ to find the eigenvectors $U$ and eigenvalues $\Omega$. We can then compute the susceptibility from Eq.(61) or, by following the pole with large weight as the momentum $\mathbf{k}$ varies in the Brillouin zone, find the dispersion relation of the collective modes. Before describing our numerical results for the soliton-lattice state, it is instructive to look at the response functions in the commensurate phase where the GRPA equations can be solved exactly. 


\section{A. Response functions in the commensurate phase}

In the commensurate phase, the only non-zero order parameter is $<\widetilde{\rho}_{R L}(0)>=1 / 2$. It follows then that

$$
\begin{aligned}
f\left(q_{x}\right) & =t_{R} \delta_{q_{x}, 0}, \\
F\left(\mathbf{q}, \mathbf{q}^{\prime}\right) & =t_{R} e^{-i \varphi} \delta_{\mathbf{q}, \mathbf{q}^{\prime}}, \\
F^{T}\left(\mathbf{q}, \mathbf{q}^{\prime}\right) & =F\left(\mathbf{q}, \mathbf{q}^{\prime}\right), \\
K\left(\mathbf{q}, \mathbf{q}^{\prime}\right) & =\frac{1}{2} e^{-i \varphi} \delta_{\mathbf{q}, \mathbf{q}^{\prime}}, \\
K^{T}\left(\mathbf{q}, \mathbf{q}^{\prime}\right) & =K\left(\mathbf{q}, \mathbf{q}^{\prime}\right),
\end{aligned}
$$

where we have defined

$$
t_{R} \equiv \widetilde{t}+\frac{1}{2} V_{d}(Q),
$$

and

$$
\varphi \equiv \frac{Q q_{y} \ell^{2}}{2} .
$$

It is very simple to solve for $\Gamma\left(\mathbf{q}, \mathbf{q}^{\prime}, \omega\right)$ given in Eq.(35). First, we use the pseudospin transformation of Eq.(51) to define a new susceptibility matrix by

$\Gamma_{p}\left(\mathbf{q}, \mathbf{q}^{\prime}, \omega\right) \equiv$

$$
\left(\begin{array}{cccc}
\chi_{n n}\left(\mathbf{q}, \mathbf{q}^{\prime}, \omega\right) & \chi_{n-}\left(\mathbf{q}, \mathbf{q}^{\prime}+\mathbf{Q}, \omega\right) & \chi_{n+}\left(\mathbf{q}, \mathbf{q}^{\prime}-\mathbf{Q}, \omega\right) & \chi_{n S_{z}}\left(\mathbf{q}, \mathbf{q}^{\prime}, \omega\right) \\
\chi_{+n}\left(\mathbf{q}+\mathbf{Q}, \mathbf{q}^{\prime}, \omega\right) & \chi_{+-}\left(\mathbf{q}+\mathbf{Q}, \mathbf{q}^{\prime}+\mathbf{Q}, \omega\right) & \chi_{++}\left(\mathbf{q}+\mathbf{Q}, \mathbf{q}^{\prime}-\mathbf{Q}, \omega\right) & \chi_{+S_{z}}\left(\mathbf{q}+\mathbf{Q}, \mathbf{q}^{\prime}, \omega\right) \\
\chi_{-n}\left(\mathbf{q}-\mathbf{Q}, \mathbf{q}^{\prime}, \omega\right) & \chi_{--}\left(\mathbf{q}-\mathbf{Q}, \mathbf{q}^{\prime}+\mathbf{Q}, \omega\right) & \chi_{-+}\left(\mathbf{q}-\mathbf{Q}, \mathbf{q}^{\prime}-\mathbf{Q}, \omega\right) & \chi_{-S_{z}}\left(\mathbf{q}-\mathbf{Q}, \mathbf{q}^{\prime}, \omega\right) \\
\chi_{S_{z} n}\left(\mathbf{q}, \mathbf{q}^{\prime}, \omega\right) & \chi_{S_{z}}\left(\mathbf{q}, \mathbf{q}^{\prime}+\mathbf{Q}, \omega\right) & \chi_{S_{z}+}\left(\mathbf{q}, \mathbf{q}^{\prime}-\mathbf{Q}, \omega\right) & \chi_{S_{z} S_{z}}\left(\mathbf{q}, \mathbf{q}^{\prime}, \omega\right)
\end{array}\right)
$$

After some simple algebra, we find

$$
\Gamma_{p}\left(\mathbf{q}, \mathbf{q}^{\prime}, \omega\right)=\left(\frac{\delta_{\mathbf{q}, \mathbf{q}^{\prime}}}{(\omega+i \delta)^{2}-\omega_{0}^{2}(\mathbf{q})}\right)\left(\begin{array}{cccc}
-2 b(1-\cos (2 \varphi) & i \omega \sin (\varphi) & -i \omega \sin (\varphi) & i b \sin (2 \varphi) \\
-i \omega \sin (\varphi) & -a & a & -\frac{\omega}{2} \cos (\varphi) \\
i \omega \sin (\varphi) & a & -a & \frac{\omega}{2} \cos (\varphi) \\
-i b \sin (2 \varphi) & -\frac{\omega}{2} \cos (\varphi) & \frac{\omega}{2} \cos (\varphi) & -\frac{b}{2}(1+\cos (2 \varphi))
\end{array}\right),
$$


where we have defined

$$
\begin{aligned}
a & =t_{R}+\frac{1}{2}\left(V_{a}(\mathbf{q})-V_{b}(\mathbf{q})-V_{c}(\mathbf{q}) \cos (2 \varphi)\right), \\
b & =t_{R}-\frac{1}{4}\left(V_{d}(\mathbf{q}+\mathbf{Q})+V_{d}(\mathbf{q}-\mathbf{Q})\right) \\
\omega_{0}^{2}(\mathbf{q}) & =4 a b .
\end{aligned}
$$

From Eq.(65), we see that the modulation of the pseudospin structure (i.e., of $\tilde{\theta}(X)$ ) due to the parallel magnetic field induces a coupling between the charge and spin response functions. In fact, as discussed in Ref. [6], this coupling is due to the magnetic field and technically arises because we have restricted the Hilbert space to the first Landau level only. In the restricted Hilbert space, pseudospin and charge operators no longer commute and it follows that modulations of the pseudospin texture are in general accompanied by modulations of the charge density. The presence of the parallel magnetic field is crucial, however, for this coupling to show up. In fact, the density response function is non-zero only in the presence of a parallel magnetic field. Without this field, the ground state consists of a fully filled Landau level of symmetric states. Since a scalar field coupling to the density does not change the pseudospin state, there is no way to excite an electron from the symmetric to the antisymmetric state. When the parallel field is added, however, symmetric and antisymmetric states are mixed and density wave excitations becomes possible. By density wave excitation, we mean here a collective excitation i.e. a pole of the two-particle Green's function.

The coupling between pseudospin and density modes is interesting because the collective excitations of the system must now be considered as mixed pseudospin and density excitations. The coupling should also be important experimentally since external electromagnetic fields will tend to couple only to the total density of the two layers; in the presence of a parallel component of the magnetic field the density-density response function has a pole at the frequency of the pseudospin collective mode. In the soliton-lattice phase to which we turn next and, which has gapless collective modes, this coupling is also present. A similar situation is encountered in spiral magnets where the non-collinearity of the spin structure gives rise to a spin-charge coupling [19]. 


\section{B. Numerical results for the collective excitations}

In the commensurate phase, the various response functions $\chi$ are very simple since they have only one real pole (no broadening). The pole of the bare response functions $\chi^{0}$ corresponds to the excitation of an electron (a particle-hole excitation) from the $E_{-}$to $E_{+}$band (Eq.(25)). When vertex corrections are introduced in the GRPA, this pole is renormalized into a dispersive collective excitation (a spin wave in the pseudospin language) and appears as a pole of $\chi$. These vertex corrections are important. To give an example, the gap calculated from $\omega_{0}(q=0)$ (Eq.(66) ), at $d / \ell=1.0$ and $t /\left(e^{2} / \epsilon_{0} \ell\right)=0.01$, is reduced by a factor six by the vertex corrections. Fig. 7 shows the dispersion relation of this spin wave, $\omega_{0}(\mathbf{q})$, for different values of the parallel magnetic field along the direction $q_{y}=0$ (the dispersion relation is not isotropic since the parallel magnetic field is set along the $y$ axis). A soft mode develops as the parallel magnetic field is increased, but the value of $Q$ at which the collective mode of the commensurate state first goes soft is larger than the value of $Q$ at which the commensurate to soliton-lattice phase transition occurs. The commensurate to soliton lattice phase transition is first order. No corresponding partial mode softening appears as a precursor of the commensurate to soliton lattice phase transition along the direction $q_{x}=0$.

The response functions of the soliton-lattice state are more interesting. Fig. 8 shows the imaginary part of the transverse response function $\chi_{+-}(\mathbf{k}+\mathbf{Q}, \mathbf{k}+\mathbf{Q}, \omega)$ (a) in the HFA and (b) in the GRPA. The renormalization of the particle-hole excitation is more dramatic than in the commensurate phase. In addition to a Goldstone mode which appears at low energies, a number of additional sharp peaks suggestive of further collective modes appear in the response functions. For wave vectors in the $\hat{x}$ direction and parallel fields close to that of the commensurate to soliton lattice phase transition, the Goldstone modes are best thought of as compressional waves of the soliton lattice. For wave vectors in the $\hat{y}$ direction the Goldstone modes in this regime are best thought of as oscillations in the position of a particular phase slip as a function of the $\hat{y}$ coordinate. This Goldstone mode is expected because of the translational invariance of the underlying Hamiltonian which is 
broken in the soliton-lattice state. The dispersion relation of the Goldstone modes is highly anisotropic as shown in Fig. 9 where it is computed for different values of $Q$ along the two perpendicular directions $q_{x}=0$ and $q_{y}=0$. Close to the phase transition between commensurate and soliton-lattice states the solitons are very widely spaced and we should expect that the velocity of the Goldstone mode in the $q_{y}=0$ direction should be very small. In this direction (the direction perpendicular to $B_{\|}$), the Goldstone mode is periodic with the periodicity of the Brillouin zone i.e. $2 \pi / L_{S}$.

As we mentioned above, in the absence of tunneling, the ground state of the system is independent of parallel field, has spontaneous inter-layer phase coherence, and has Goldstone modes corresponding to this broken symmetry. The incommensurate state is the HartreeFock approximation for the broken symmetry state. For non-zero values of the tunneling amplitude in a parallel field, either the commensurate or the soliton-lattice state has a lower energy and the phase transition between these states occurs at a critical parallel field. For very strong magnetic fields, however, the soliton-lattice state asymptotically approaches the incommensurate state. The Goldstone modes of the soliton-lattice state, which are most naturally associated with broken translational symmetry, must crossover to the Goldstone modes of the spontaneous-phase-coherence state. These are easily obtained by solving GRPA equations at $t=0$. (The calculations are very similar to those for the commensurate state). We find immediately that the response functions have a single pole at a frequency given by

$$
\omega_{s c}(q)=\sqrt{\left[V_{a}(q)-V_{b}(q)-V_{c}(q)+V_{d}(0)\right]\left[V_{d}(0)-V_{d}(q)\right]} .
$$

In Fig. 10 we compare the collective modes of the soliton-lattice state in the strong-field regime (where $Q_{S} \approx Q$ ) with the collective modes of the spontaneously phase-coherent state in the absence of tunneling. As expected the collective modes are nearly identical. For the soliton-lattice state many collective modes appear at each wavevector; for $\left(k_{x}, k_{y}\right)$ the energies are close to $\omega_{s c}\left(\left|\left(k_{x}+n Q, k_{y}\right)\right|\right)$ where $n$ is an integer. (The higher energy collective modes in Fig. 8 correspond to $n \neq 0$.) The broken translational symmetry of the soliton lattice state results in a folding of the collective modes of the $t=0$ ground state. At the 
parallel field of Fig. 10 mode-mode coupling effects due to the interlayer hopping are weak; as the parallel field weakens and the phase transition is approached, the coupling becomes stronger and eventually the modes become more similar to those of the incommensurate state, except at wavelengths approaching the lengthening soliton-lattice period.

At strong parallel fields, the effect of the tunneling on the spontaneously-coherent ground state is very much like the effect of a grating with wavevector $Q$. We are presently investigating the possibility [21] of using tunneling in a parallel field as a tunable grating which allows light to couple to finite wave vector modes of the spontaneously phase-coherent state in much the same way that artificially generated metallic gratings are used to couple to finite wave vector plasmons of two-dimensional electron systems. [20]

Finally, we have verified that the pseudospin-charge coupling that we discussed in the commensurate phase also appears in the soliton phase. However, the pseudospin-charge coupling is very small. Typically, the density response function is more than one-thousand times smaller than the pseudospin response functions $\chi_{Z Z}$ or $\chi_{+-}$. The weight of the Goldstone mode in the density response function (as in all other response functions) vanishes as the wave vector of the excitation vanishes. Further studies will be needed to understand how this coupling affects the ground-state conductivity of the soliton lattice.

\section{CONCLUSION}

We have studied the commensurate-incommensurate phase transition which appears in a DQWS in a strong magnetic field when the field is tilted away from the normal to the planes of the two-dimensional electron gas in each well. Working in the Hartree-Fock approximation, we have calculated the energy of the soliton-lattice state for different values of the tunneling parameter and parallel magnetic field and found that the soliton-lattice state is the ground-state of the system for all angles greater than the critical tilt angle. The difference in energy between the soliton-lattice state and the incommensurate state is however very small. Close to the commensurate-soliton lattice transition, we find that the gradient 
approximation described in Ref. [8] is a good approximation for the soliton-lattice energy and for the modulation of the interlayer coherence phase. This approximation soon breaks down, however, as the parallel magnetic field is increased.

Using the GRPA, we have also studied the collective modes of the soliton lattice. As expected from the broken translational symmetry of the soliton lattice, there is a Goldstone mode branch which represents the phonons of the soliton lattice. Because of the one-dimensional character of the Brillouin zone, the Goldstone mode dispersion relation is periodic only in the direction perpendicular to the parallel magnetic field. Beside the Goldstone mode, there are also higher-energy collective excitations in the response functions calculated in the GRPA. In the limit of strong parallel magnetic fields, we were able to relate these higher-energy excitations to the collective modes of the spontaneously-coherent state in the absence of tunneling at wavevectors displaced by multiples of $Q \hat{x}$. In this regime, the effect of tunneling in a parallel field resembles that of a finite-wave-vector grating which mixes the different modes of the spontaneously coherent state.

Finally, we have also shown that, except in the direction perpendicular to the parallel magnetic field, the collective modes in the system are in fact mixed pseudospin-charge excitations. Since all response functions are coupled in the soliton lattice, it follows that the den-

sity response function has poles at exactly the frequency of these mixed pseudospin-charge excitations (including the Goldstone mode). More work is needed, however, to understand how this coupling could affect the conductivity of the soliton-lattice state.

\section{ACKNOWLEDGEMENT}

This research was supported in part by NATO Collaborative Research Grant No. 930684. RC acknowledges support from the Natural Sciences and Engineering Research Council of Canada (NSERC) and from the Fonds pour la formation de chercheurs et l'aide à la recherche from the Government of Québec (FACR). HF acknowledges support from NSF grant No. DMR 92-02255, from the Sloan Foundation, and from the Research Corporation. 
LB acknowledges support from CICyT of Spain under contract No. MAT 91-0201. AHM acknowledges support from NSF grant No. DMR 94-16906. The authors acknowledge helpful discussions with Kun Yang and Steve Girvin. 


\section{REFERENCES}

[1] See for example, Y.W. Suen et al., Phys. Rev. Lett. 68, 1379 (1992); J.P. Eisenstein et al., Phys. Rev. Lett. 68, 1383 (1992).

[2] See for example, B. I. Halperin, Helv. Phys. Acta 56, 75 (1983); A.H. MacDonald, Surf. Sci. 229, 1 (1990); Z. F. Ezawa and A. Iwazaki, Phys. Rev. B 47, 7295 (1993); Song He et al., Phys. Rev. B 47, 4394 (1993); T. Chakraborty and P. Pietiläinen, Phys. Rev. Lett. 59, 2784 (1987); E.H. Rezayi and F.D.M. Haldane, Bull. Am. Phys. Soc. 32, 892 (1987); D. Yoshioka, A.H. MacDonald, and S.M. Girvin, Phys. Rev. B 39, 1932 (1989).

[3] X.G. Wen and A. Zee, Phys. Rev. Lett. 69, 1811 (1992); X.G. Wen and A. Zee, Phys. Rev. B 47, 2265 (1993).

[4] Z.F. Ezawa and A. Iwazaki, J. Mod. Phys. B 19, 3205 (1992).

[5] H. Fertig, Phys. Rev. B40, 1087 (1989); A.H. MacDonald, P.M. Platzman, and G.S. Boebinger, Phys. Rev. Lett. 65, 775 (1990); L. Brey, ibid. 65, 903 (1990); X.M. Chen and J.J. Quinn, Phys. Rev. B 45, 11054 (1992).

[6] K. Moon, H. Mori, K. Yang, S. M Girvin, A. H. MacDonald, L. Zheng, D. Yoshioka and S.C. Zhang, submitted to Phys. Rev. B (1994).

[7] S. Q. Murphy, J. P. Eisenstein, G. S. Boebinger, L. N. Pfeiffer, and K. W. West, Phys. Rev. Lett. 72, 728 (1994).

[8] K. Yang et al.,Phys. Rev. Lett. 72, 732 (1994).

[9] K. Moon, H. Mori, K. Yang, S. M Girvin, A. H. MacDonald, L. Zheng, D. Yoshioka and S.C. Zhang, submitted to Phys. Rev. B (1994).

[10] P. Bak, Rep. Prog. Phys. 45, 587 (1982).

[11] George Gruner, Density Waves in Solids, (Addison-Wesley, Reading, 1994). 
[12] R. Côté and A. H. MacDonald, Phys. Rev. Lett. 65, 2662 (1990); Phys. Rev. B 44, 8759 (1991).

[13] R. Côté, L. Brey and A. H. MacDonald, Phys. Rev. B46, 10239 (1992).

[14] J. Hu and A. H. MacDonald, Phys. Rev. B 46, 12554 (1992).

[15] In the general case we can define a Fourier transform which depends on two wavevectors. See Refs. 12 and 13.

[16] Kun Yang and A.H. MacDonald, submitted to Phys. Rev. B. (1994).

[17] S. L. Sondhi, A. Karlhede, S. A. Kivelson, E. H. Rezayi, Phys. Rev. B 47, 16419 (1993).

[18] H. A. Fertig, L. Brey, R. Côté and A. H. MacDonald, , Phys. Rev. B. 50, 11018 (1994).

[19] R. Côté and A.-M. S. Tremblay, accepted for publication in Europhysics Letters (1994).

[20] See for example, D. Heitmann, Surf. Sci. 170, 332 (1986); J.P. Kotthaus, in Interfaces, Quantum Wells, and Superlattices, edited by C.R. Leavens, and R. Taylor (Plenum, New York, 1988), p. 95; and D. Heitmann, in Physics and Applications of Quantum Wells, and Superlattices, edited by K. v. Klitzing and E.E. Mendez (Plenum, New York, 1988), p. 317. For a recent discussion of the theory of the electromagnetic coupling between a grating and a nearby two-dimensional electron system see Lian Zheng, W.L. Schaich, and A.H. MacDonald, Phys. Rev. B 41, 8439 (1990).

[21] R. Côté, A. H. MacDonald, H. Fertig and L. Brey, work in progress. 


\section{FIGURES}

FIG. 1. Energy of the commensurate, incommensurate and soliton-lattice states as a function of $Q \ell$ for $(\mathrm{a}) d / \ell=1.0, t /\left(e^{2} / \epsilon_{0} \ell\right)=0.01$ and $(\mathrm{b}) d / \ell=1.877, t /\left(e^{2} / \epsilon_{0} \ell\right)=0.005$. The dotted line corresponds to the energy of the commensurate phase calculated in the gradient approximation.

FIG. 2. Behavior of $\widetilde{\theta}(X)$ over one period of the soliton lattice in the HFA. (a) $d / \ell=1.0, t /\left(e^{2} / \epsilon_{0} \ell\right)=0.01, Q \ell=0.63$ (just after the $C \rightarrow S$ transition); (b) $d / \ell=1.0, t /\left(e^{2} / \epsilon_{0} \ell\right)=0.01, Q \ell=1.5$ (in the high parallel magnetic field limit). The dotted lines represent the sine-Gordon solution for $\tilde{\theta}(X)$

FIG. 3. Behavior of the ratio $Q_{S} / Q$ (soliton wave vector to parallel magnetic field wave vector) as a function of $Q \ell$ for $d / \ell=1.0, t /\left(e^{2} / \epsilon_{0} \ell\right)=0.01$. The line at $Q_{S} / Q=1$ is a guide to the eyes.

FIG. 4. Shape of the upper band $E_{+}(X)$ over one period of the soliton lattice in the HFA for (a) $d / \ell=1.0, t /\left(e^{2} / \epsilon_{0} \ell\right)=0.01, Q \ell=0.63$ and (b) $d / \ell=1.0, t /\left(e^{2} / \epsilon_{0} \ell\right)=0.01, Q \ell=1.5$.

FIG. 5. Energy of the commensurate state as a function of $Q \ell$ for different values of the bare hopping parameter $t$. (1) $t /\left(e^{2} / \epsilon_{0} \ell\right)=0.1 ;(2) t /\left(e^{2} / \epsilon_{0} \ell\right)=0.01 ;(3) t /\left(e^{2} / \epsilon_{0} \ell\right)=0.001$.

FIG. 6. Critical tilt angle at which the transition from commensurate to incommensurate states appears as a function of the bare hopping for $d / \ell=1.0$. The full line is a fit to a square root dependence.

FIG. 7. Dispersion relation of the spin wave mode in the commensurate phase for $d / \ell=1.0, t /\left(e^{2} / \epsilon_{0} \ell\right)=0.01$ and different values of the parallel magnetic field.

FIG. 8. Imaginary part of $\chi_{+-}(\mathbf{k}+\mathbf{Q}, \mathbf{k}+\mathbf{Q}, \omega)$ for $d / \ell=1.0, t /\left(e^{2} / \epsilon_{0} \ell\right)=0.01, Q \ell=0.72$ and wave vectors $\mathbf{k} \ell=(0.1,0),(0.3,0)$. (a) HFA and (b) GRPA. The Goldstone modes appear as low-energy peaks in (b). 
FIG. 9. Dispersion relations of the Goldstone mode at (a) $d / \ell=1.0, t /\left(e^{2} / \epsilon_{0} \ell\right)=0.01$, $Q \ell=0.72$, and $(\mathrm{b}) d / \ell=1.0, t /\left(e^{2} / \epsilon_{0} \ell\right)=0.01, Q \ell=1.5$ along the directions $k_{x}=0$ and $k_{y}=0$. The dispersion relation is highly anisotropic, being in fact periodic only along $k_{y}=0$.

FIG. 10. Comparison between the collective modes of the soliton lattice (•) at, $t /\left(e^{2} / \epsilon_{0} \ell\right)=0.01$, in the high-parallel magnetic field regime and the collective modes of the spontaneously-coherent state in the absence of tunneling (lines). The parameters are $d / \ell=1.0$, $Q \ell=1.5$ and $k_{y}=0$. For the soliton lattice, only the strongest six modes in the response function $\chi_{+-}$are shown. For the spontaneously-coherent state, $\omega_{s c}\left(k_{x}+n Q\right)$ is plotted for $n=-2,-1, \ldots, 4$. The dotted line represents $\omega_{s c}\left(k_{x}\right)$. 


\section{APPENDIX: THE GRADIENT APPROXIMATION}

In this appendix, we derive the Hartree-Fock approximation equation of motion for the single-particle Green's functions $G_{i, j}(X, \tau)=-<T c_{i, X}(\tau) c_{j, X}^{\dagger}(0)>$ instead of for its Fourier transform as in Eq.(9). We then introduce the so-called gradient approximation developed in Ref. [8]. This approximation is very helpful in understanding how the system evolves from the commensurate to incommensurate phases in the presence of a parallel magnetic field.

In $X$ space, the Hartree-Fock Hamiltonian of Eq.(8) is

$$
\begin{aligned}
H & =-\tilde{t} \sum_{X}\left(e^{-i Q X} \rho_{R L}(X)+e^{i Q X} \rho_{L R}(X)\right) \\
& -\frac{1}{g}\left(\frac{e^{2}}{\epsilon_{0} \ell}\right) \sum_{X, X^{\prime}} K\left(X-X^{\prime}\right)\left[<\rho_{R L}(X)>\rho_{L R}\left(X^{\prime}\right)+<\rho_{L R}(X)>\rho_{R L}\left(X^{\prime}\right)\right],
\end{aligned}
$$

where we again assume that the DQWS is unpolarized and neglect the constant first term in Eq.(8). We have also defined the operator

$$
\rho_{i, j}(X)=c_{i, X}^{\dagger} c_{j, X}
$$

and the function

$$
K\left(X-X^{\prime}\right)=\sum_{q_{x}} V_{d}\left(q_{x}\right) \cos \left[q_{x}\left(X-X^{\prime}\right)\right]
$$

The equations of motion for the single-particle Green's functions $G_{i, j}(X, \tau)$ are

$$
\begin{aligned}
\left(i \omega_{n}+\mu\right) G_{R R}\left(X, \omega_{n}\right)+S^{*}(X) G_{L R}\left(X, \omega_{n}\right) & =1 \\
\left(i \omega_{n}+\mu\right) G_{L R}\left(X, \omega_{n}\right)+S(X) G_{R R}\left(X, \omega_{n}\right) & =0
\end{aligned}
$$

where

$$
J(X)=\frac{1}{g}\left(\frac{e^{2}}{\epsilon_{0} \ell}\right) \sum_{X^{\prime}} K\left(X-X^{\prime}\right)<\rho_{R L}\left(X^{\prime}\right)>,
$$

and

$$
S(X)=\widetilde{t} e^{i Q X}+J(X)
$$

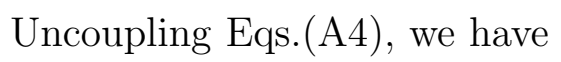




$$
G_{R R}\left(X, \omega_{n}\right)=\frac{\frac{1}{2}}{i \omega_{n}+\mu-|S(X)|}+\frac{\frac{1}{2}}{i \omega_{n}+\mu+|S(X)|},
$$

and

$$
G_{L R}\left(X, \omega_{n}\right)=\frac{1}{2}\left(\frac{S(X)}{|S(X)|}\right)\left[\frac{-1}{i \omega_{n}+\mu-|S(X)|}+\frac{1}{i \omega_{n}+\mu+|S(X)|}\right] .
$$

There are two bands with energies $\pm|S(X)|$. At $T=0 K$, one of these bands must be entirely filled with the other one empty in order to insure that the filling factor of the DQWS be exactly one. Thus,

$$
<\rho_{R L}(X)>=\frac{1}{2}\left(\frac{S(X)}{|S(X)|}\right) \equiv \frac{1}{2} e^{i \theta(X)},
$$

or,

$$
\tan \theta(X)=\frac{\Im[S(X)]}{\Re[S(X)]}
$$

This is the Hartree-Fock equation for the order parameter in $X$ space. We have devised an iterative scheme to solve this equation and at the same time minimize the Hartree-Fock ground-state energy per particle which is given by

$$
E=-\frac{\widetilde{t}}{g} \sum_{X} \cos [\theta(X)-Q X]-\frac{1}{4 g^{2}}\left(\frac{e^{2}}{\epsilon_{0} \ell}\right) \sum_{X, X^{\prime}} K\left(X-X^{\prime}\right) \cos \left(\theta(X)-\theta\left(X^{\prime}\right)\right) .
$$

We obtain results which are identical to those obtained using the approach described in the body of this paper.

It is easy to see that Eq. (A10) can be obtained by minimizing the energy with respect to the phase i.e. with $\frac{\delta E}{\delta \theta(X)}=0$. The Hartree-Fock solution is thus an extremum of the Hartree-Fock energy as expected.

The function $K\left(X-X^{\prime}\right)$ is a rapidly decreasing function of $\left|X-X^{\prime}\right|$. If the angle $\theta(X)$ does not vary too rapidly in space, this suggests that we can expand the cosine function in Eq.(A11). We get in this way

$$
E \simeq-\frac{1}{4}\left(\frac{e^{2}}{\ell}\right) V_{d}(0)-\frac{\tilde{t}}{g} \sum_{X} \cos [\theta(X)-Q X]+\frac{\gamma}{8 g^{2}} \sum_{X}\left(\frac{d \theta(X)}{d X}\right)^{2},
$$


where $\gamma=\left(\frac{e^{2}}{\epsilon_{0} \ell}\right) \sum_{X} K(X) X^{2}$. Going to the continuum limit with $\sum_{X} \ldots \longrightarrow \frac{L_{y}}{2 \pi \ell^{2}} \int d X \ldots$, we have finally for the total energy of the system:

$$
E_{T} \simeq-\frac{g}{4}\left(\frac{e^{2}}{\ell}\right) V_{d}(0)+L_{y} \int d X\left[\frac{1}{2} \rho_{S}\left(\frac{d \widetilde{\theta}(X)}{d X}+Q\right)^{2}-\frac{\widetilde{t}}{2 \pi \ell^{2}} \cos (\tilde{\theta}(X))\right]
$$

where $\tilde{\theta}(X)=\theta(X)-Q X$ and

$$
\begin{aligned}
\rho_{S} & =\left(\frac{e^{2}}{\epsilon_{0} \ell}\right) \frac{L_{y}}{16 g \pi^{2} \ell^{4}} \int d X K(X) X^{2}, \\
& =-\left.\left(\frac{e^{2}}{\epsilon_{0} \ell}\right) \frac{1}{8 \pi \ell^{2}} \frac{d^{2} V_{d}(Q)}{d Q^{2}}\right|_{Q=0} .
\end{aligned}
$$

If we now minimizes this total energy with respect to $\widetilde{\theta}(X)$, we find the well-known sine-Gordon equation

$$
\frac{d^{2} \tilde{\theta}(X)}{d X^{2}}=\frac{\tilde{t}}{2 \pi \rho_{S} \ell^{2}} \sin (\tilde{\theta}(X))
$$

which admits the kink soliton as a solution:

$$
\tilde{\theta}(X)=4 \tan ^{-1}\left[e^{-\sqrt{\frac{\widetilde{t}}{2 \pi \rho_{S} \ell^{2}}} X}\right] .
$$

If we insert Eq.(A16) in Eq.(A13), we find that the energy per particle in the presence of a single soliton is

$$
E_{S S} \simeq E_{C}+L_{y} \rho_{S}\left[8 \sqrt{\frac{\tilde{t}}{2 \pi \rho_{S} \ell^{2}}}-2 \pi Q\right]
$$

The first term in the right-hand-side of this equation is the energy per particle in the commensurate phase given in Eq.(15) while the second term is the change in this energy due to the presence of the soliton. When this second term becomes less than zero, we expect a phase transition in the system to occur since then the energy to create one soliton becomes negative. This happens at a parallel magnetic field given by

$$
B_{\left.\|_{(C \rightarrow S}\right)}=B_{\perp}\left(\frac{2 l}{\pi d}\right) \sqrt{\frac{2 \widetilde{t}}{\pi \rho_{S}}} .
$$

If we define the width of the soliton as 


$$
\xi=\frac{1}{\sqrt{\frac{\widetilde{t}}{2 \pi \rho_{S} \ell^{2}}}}
$$

then, just at the transition, we have

$$
\xi_{c}=\frac{4}{\pi Q}
$$

The critical magnetic field given in Eq.(A18) should be compared with the critical field for the transition from the commensurate to the incommensurate phase. In the gradient approximation, this critical field is given by given by

$$
B_{\|(C \rightarrow I)}=\left(\frac{\pi}{2 \sqrt{2}}\right) B_{\perp}\left(\frac{2 \ell}{\pi d}\right) \sqrt{\frac{2 \widetilde{t}}{\pi \rho_{S}}} .
$$

Finally, for a periodic ground state with regularly spaced solitons, the number of solitons is given by $N_{S}=L_{x} / L_{S}$ where $L_{S}$ is the distance between two solitons. If we neglect the soliton-soliton interaction, then the energy of the configuration is approximately given by

$$
E \simeq E_{C}+\rho_{S}\left(Q_{S} \ell\right)[8 \xi \ell-2 \pi Q \ell]
$$

where $Q_{S}=2 \pi / L_{S}$. For $Q>4 /(\pi \xi)$ this energy is always lowered by increasing $Q_{S}$ and hence the density of solitons. Repulsive interactions between the solitons must be included to determine the equilibrium value of $Q_{S}$. 


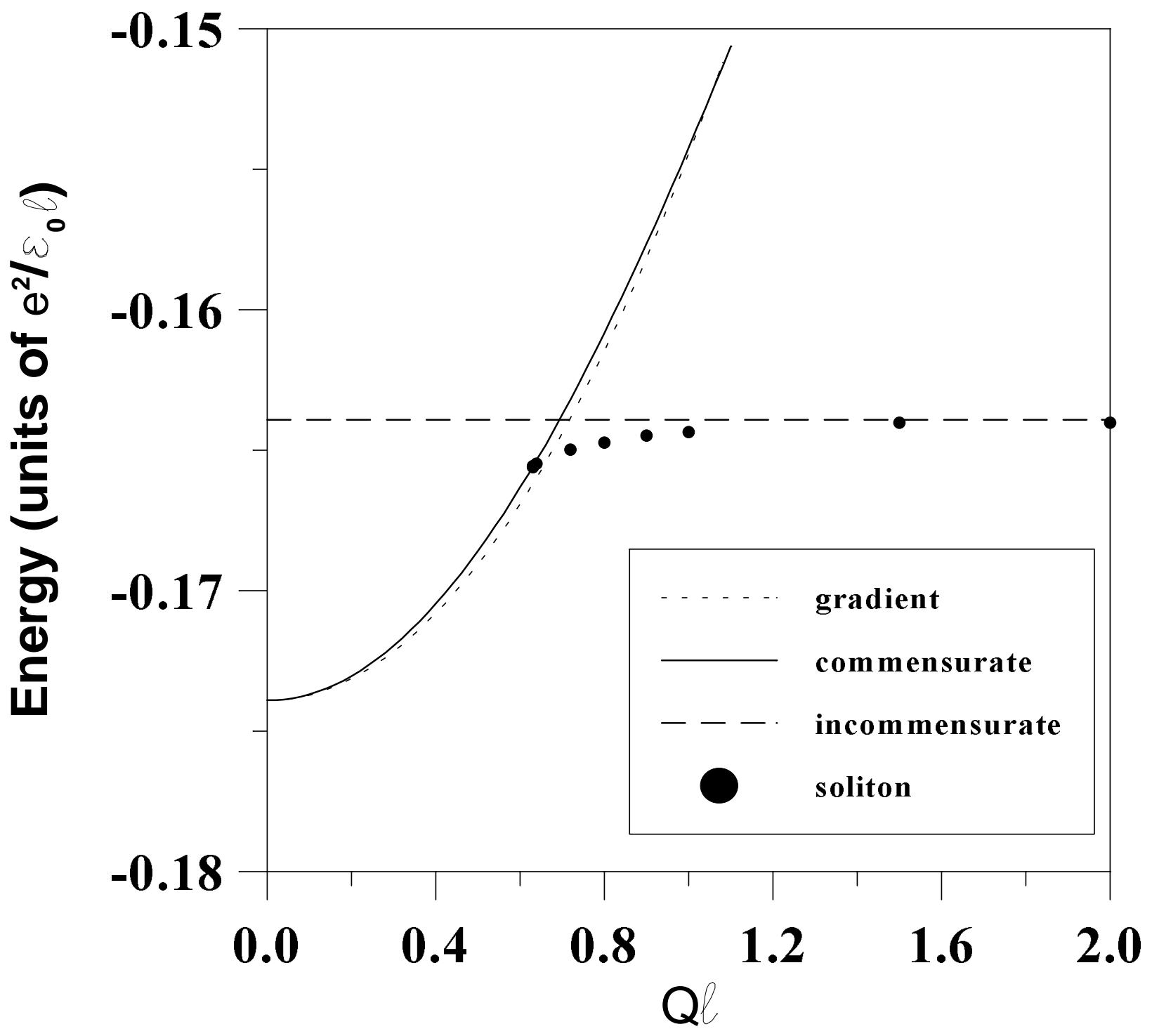

Fig. 1(a), R. Côté et al. 


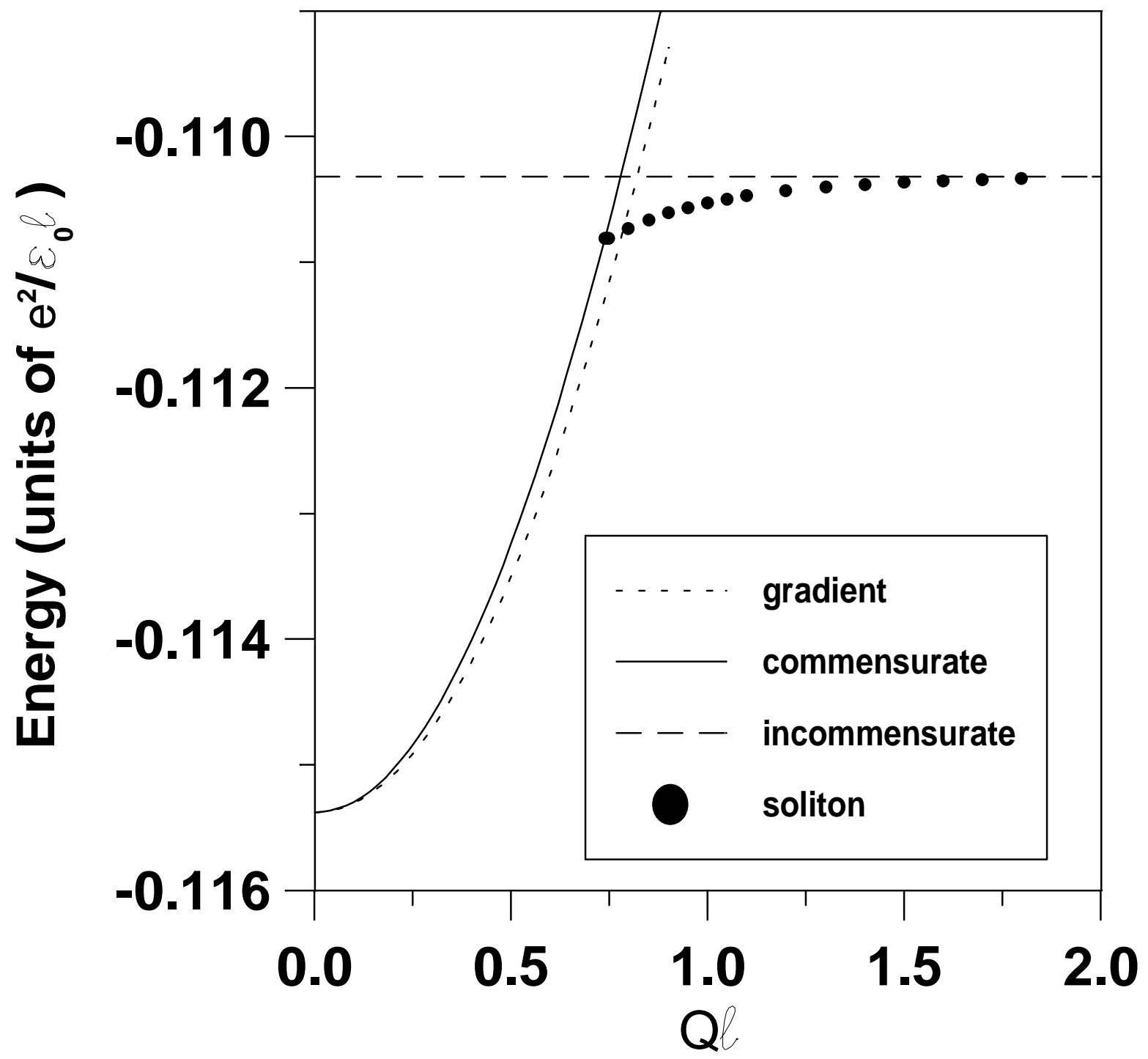

Fig. 1(b), R. Côté et al. 


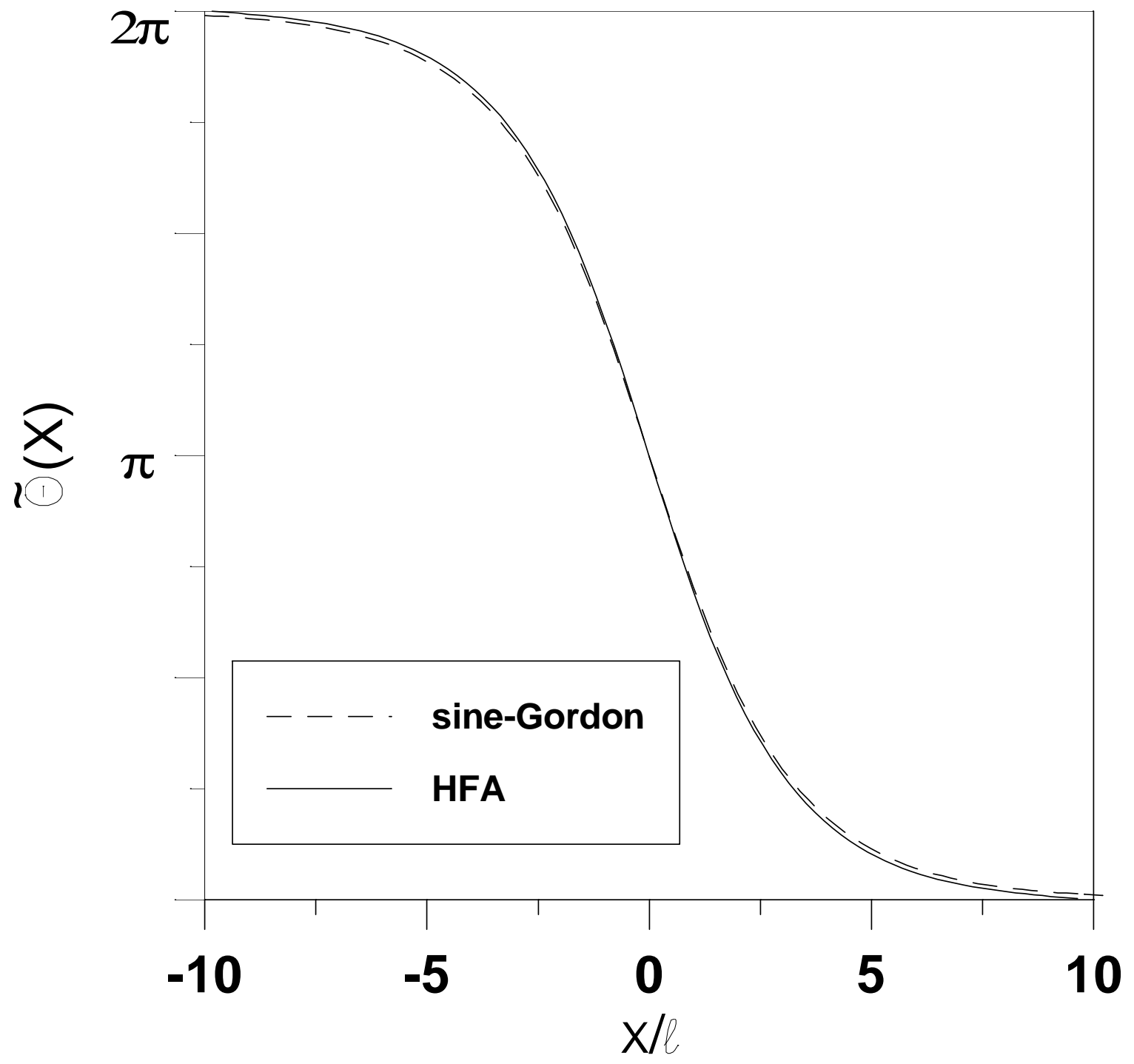

Fig. 2(a), R. Côté et al. 


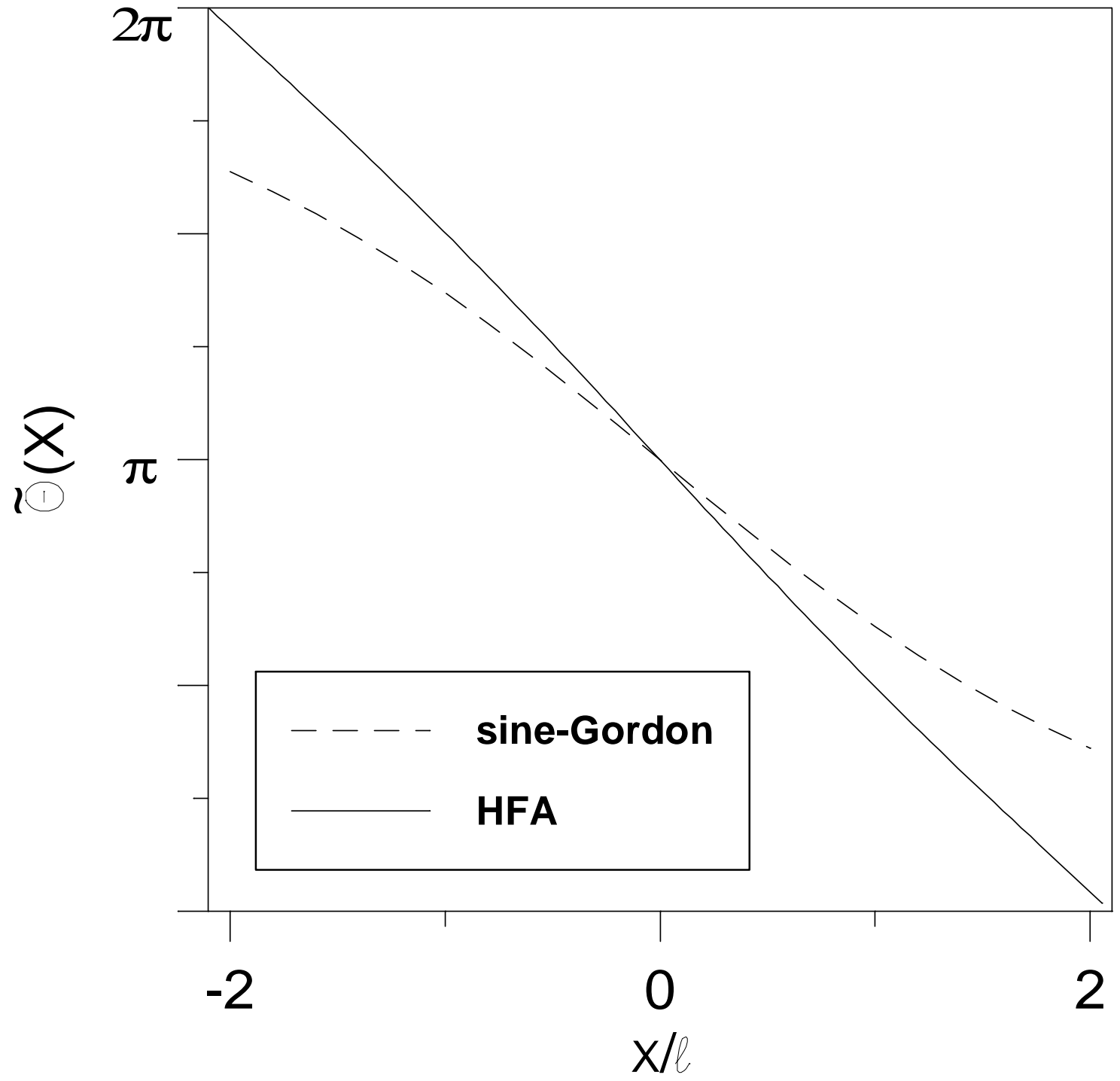

Fig. 2(b) R. Côté et al. 


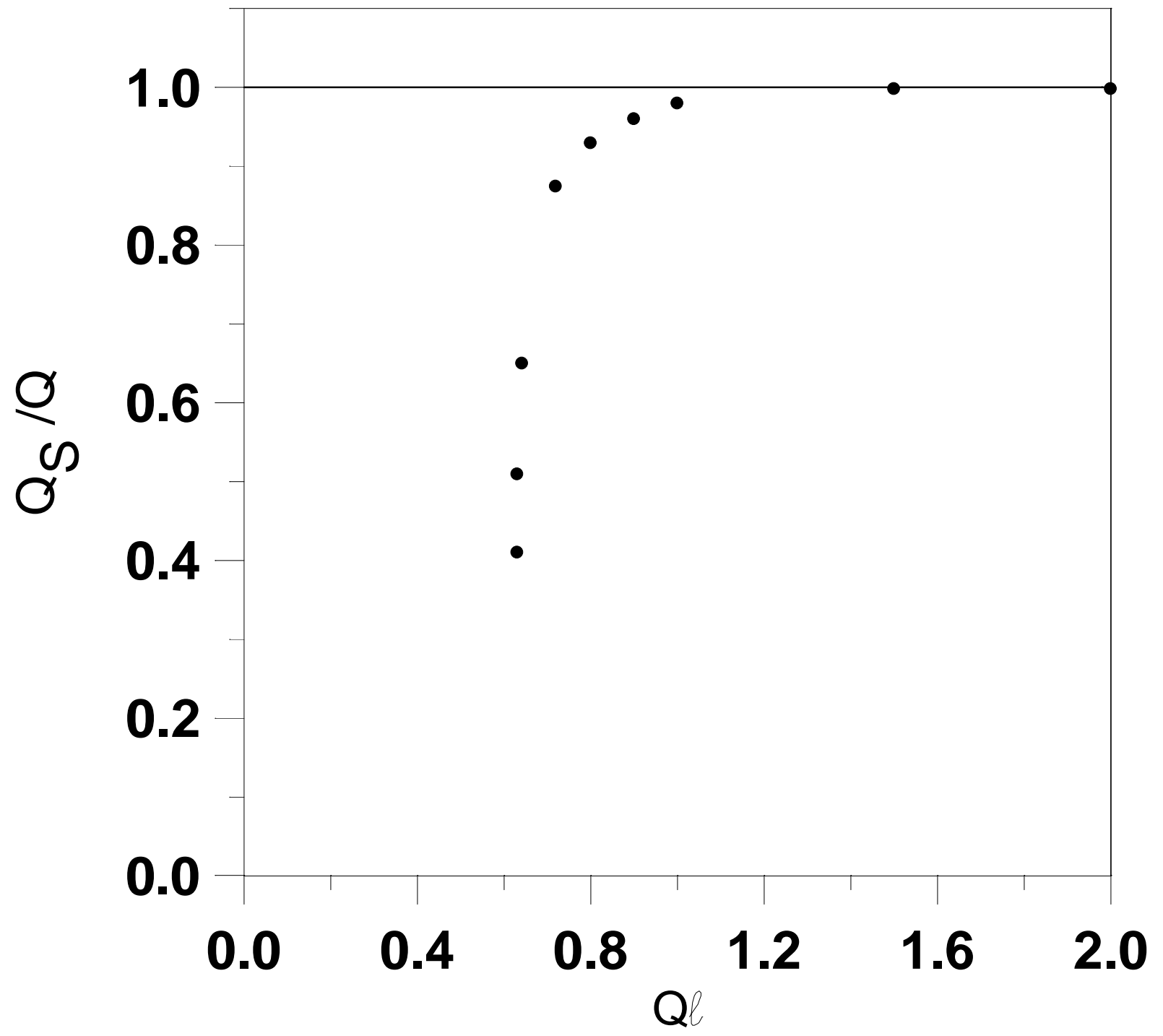

Fig. 3 R. Côté et al. 


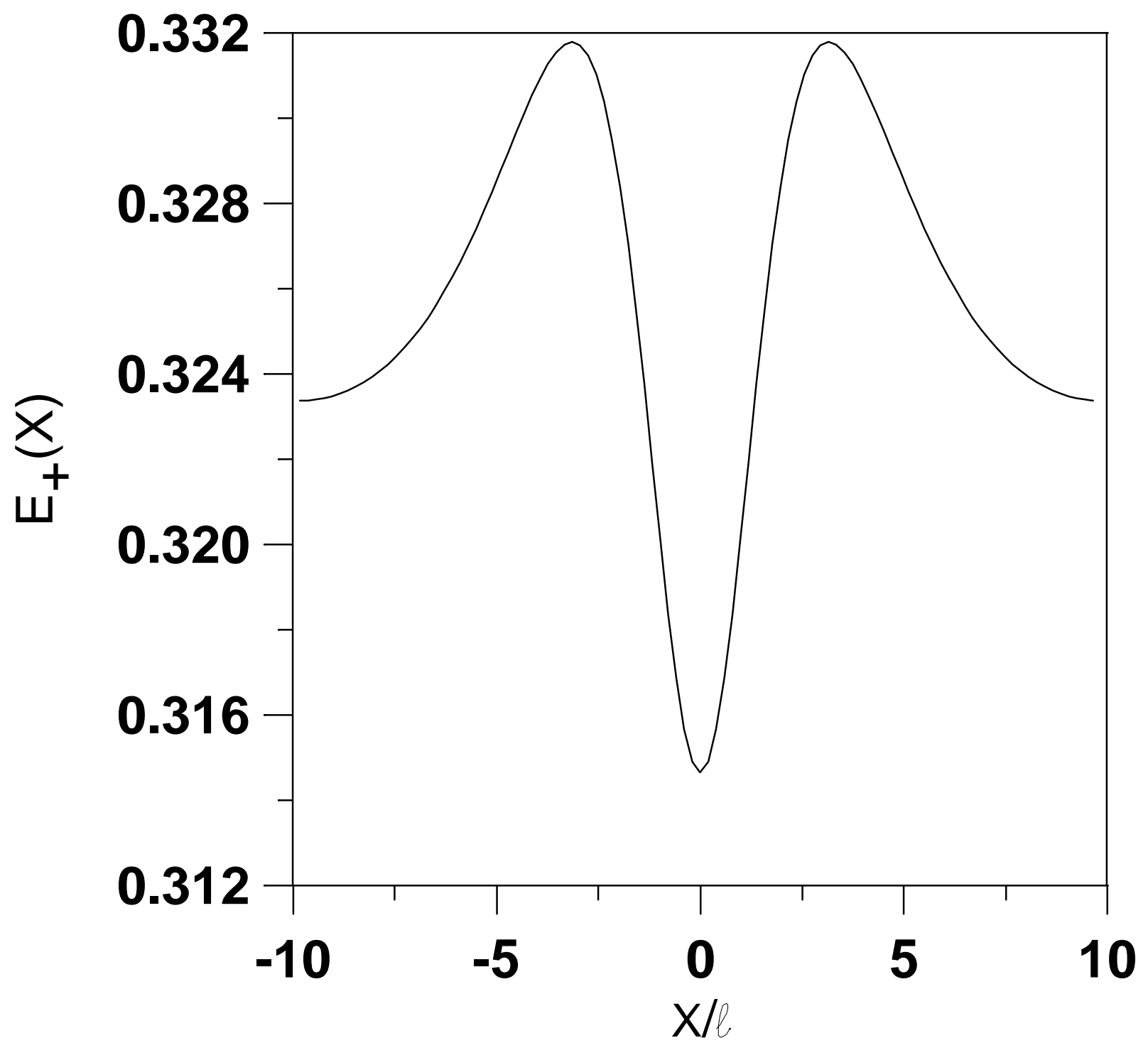

Fig. 4(a) R. Côté et al. 


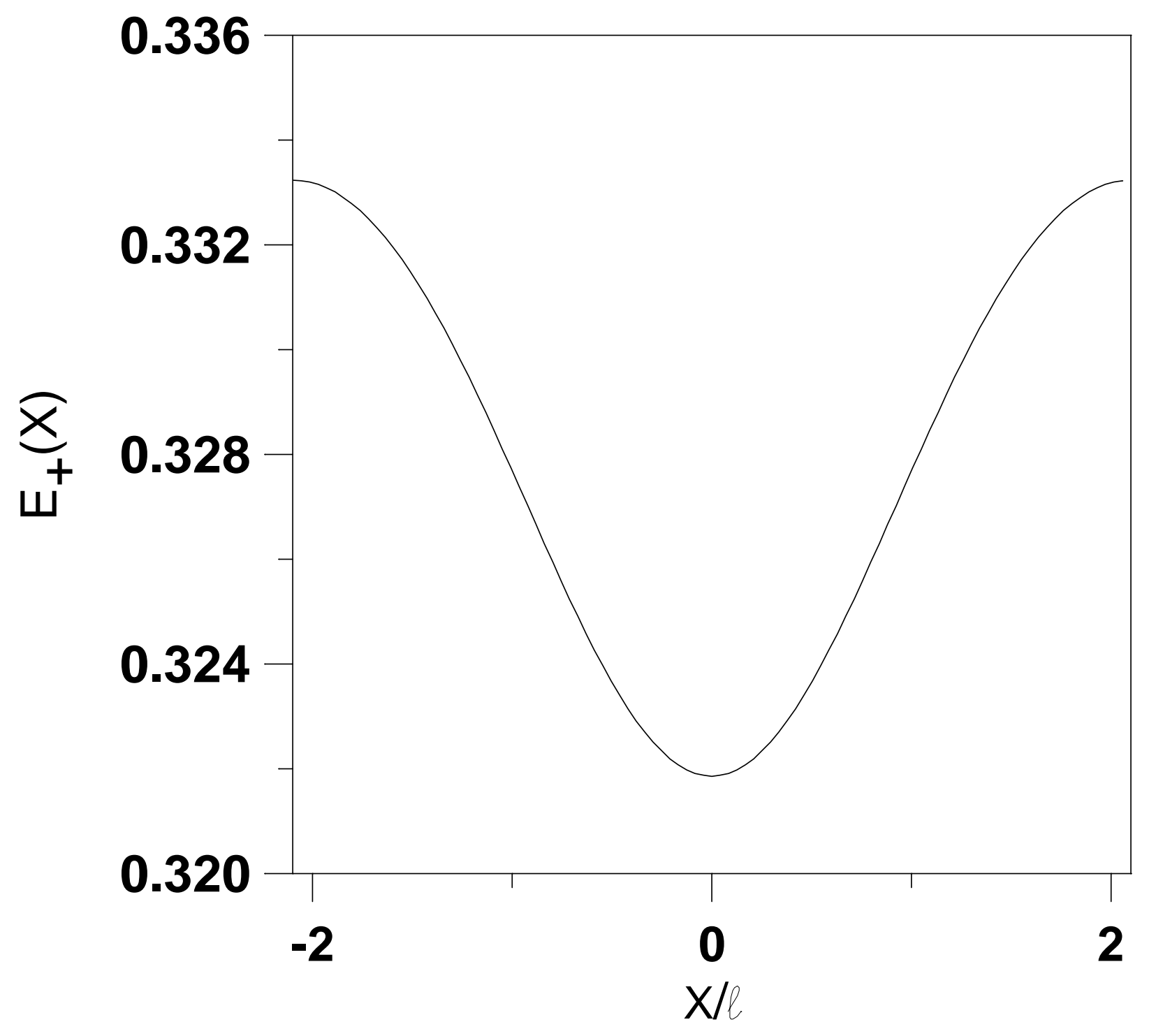

Fig. 4(b) R. Côté et al. 


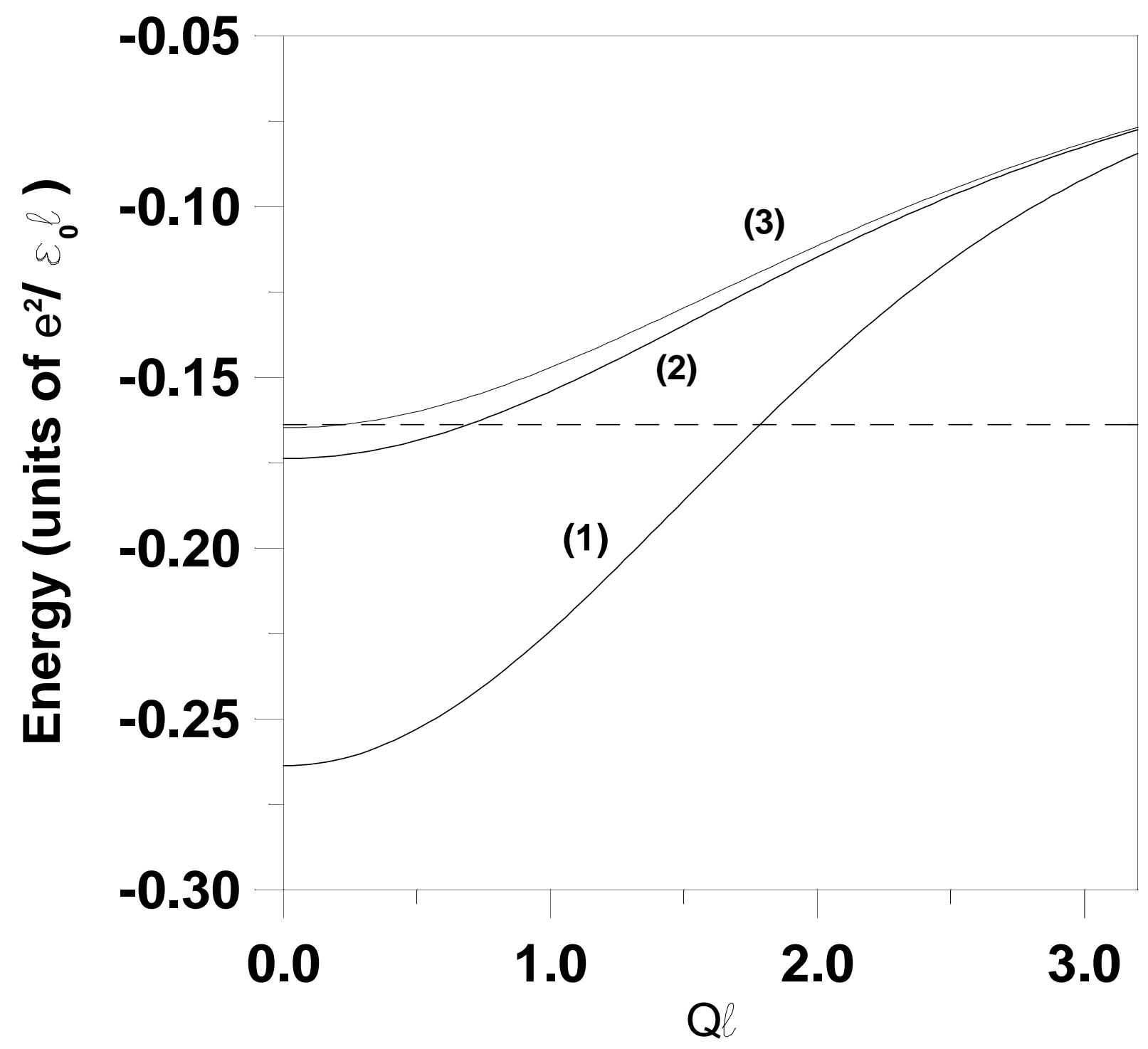

Fig. 5 R. Côté et al. 


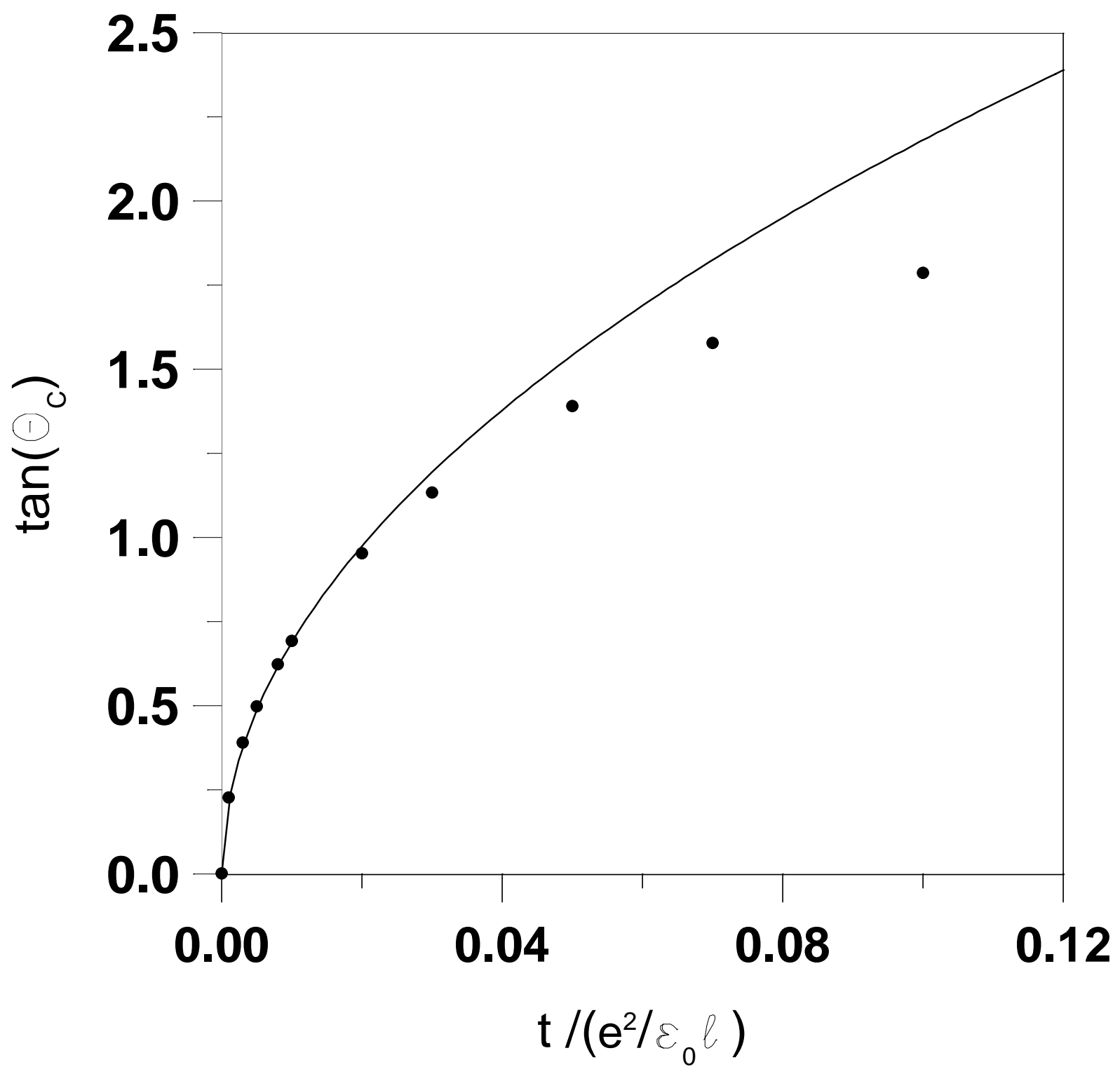

Fig. 6 R. Côté et al. 


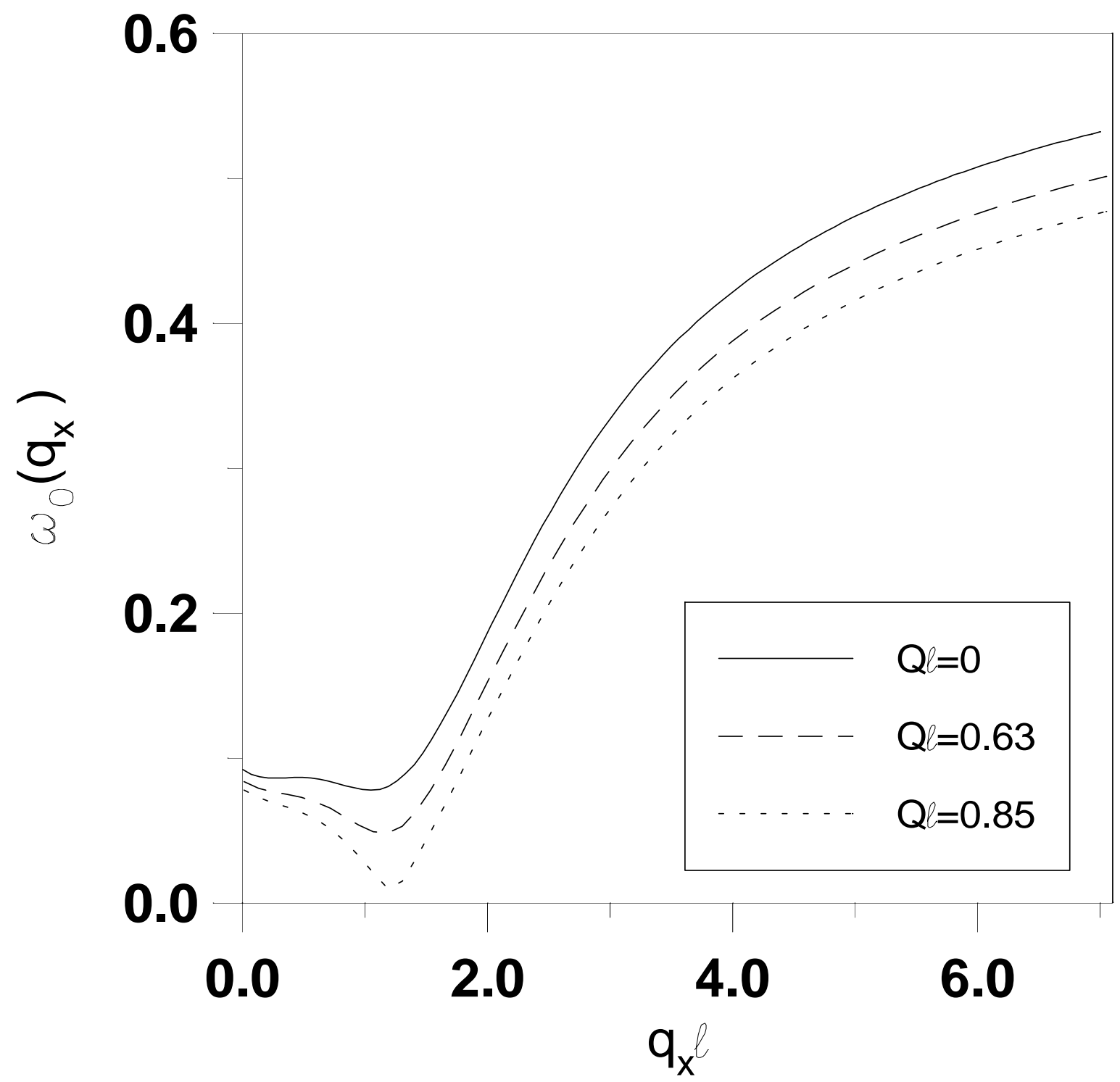

Fig. 7 R. Côté et al. 


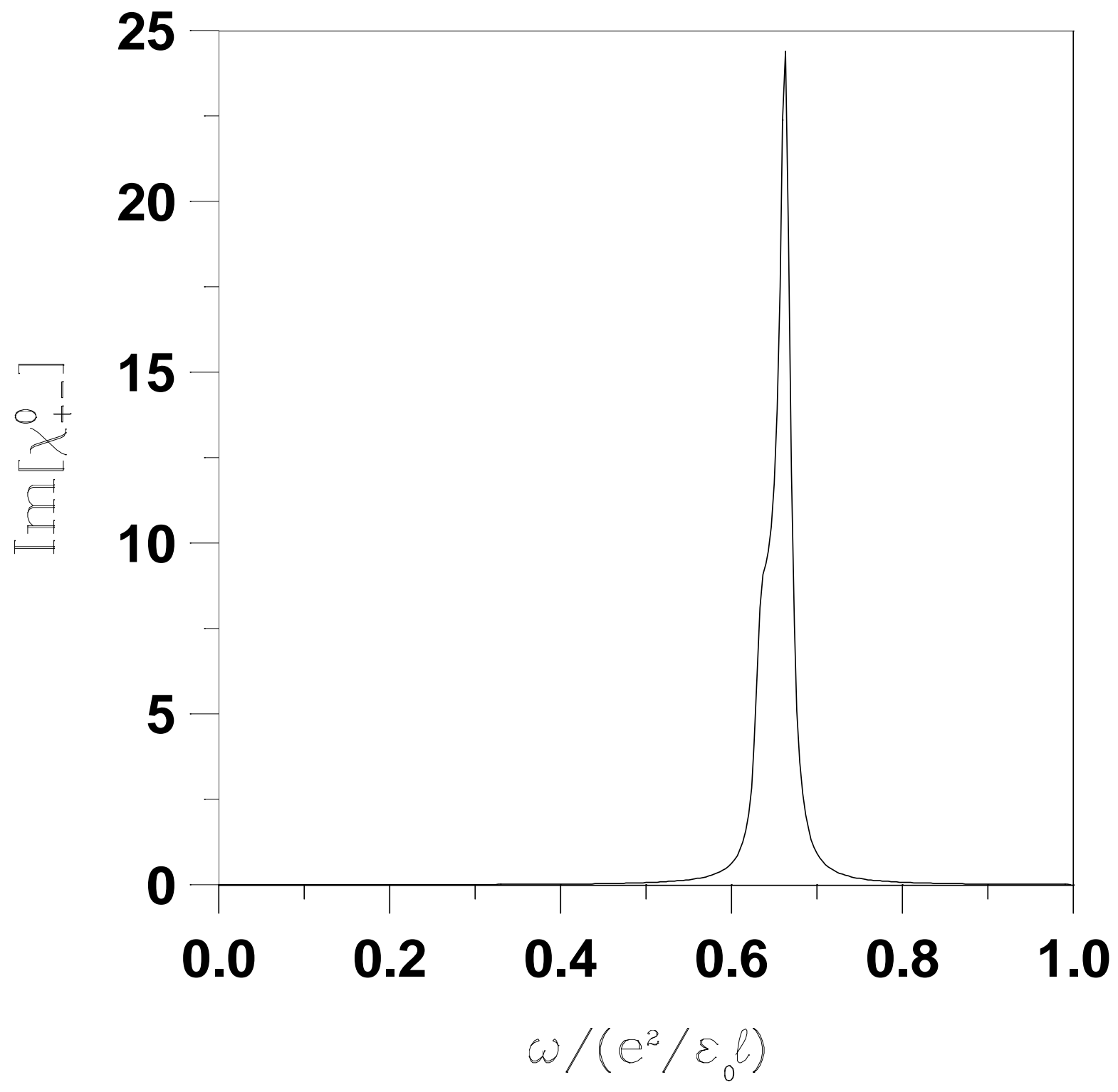

Fig. 8(a) R. Côté et al. 


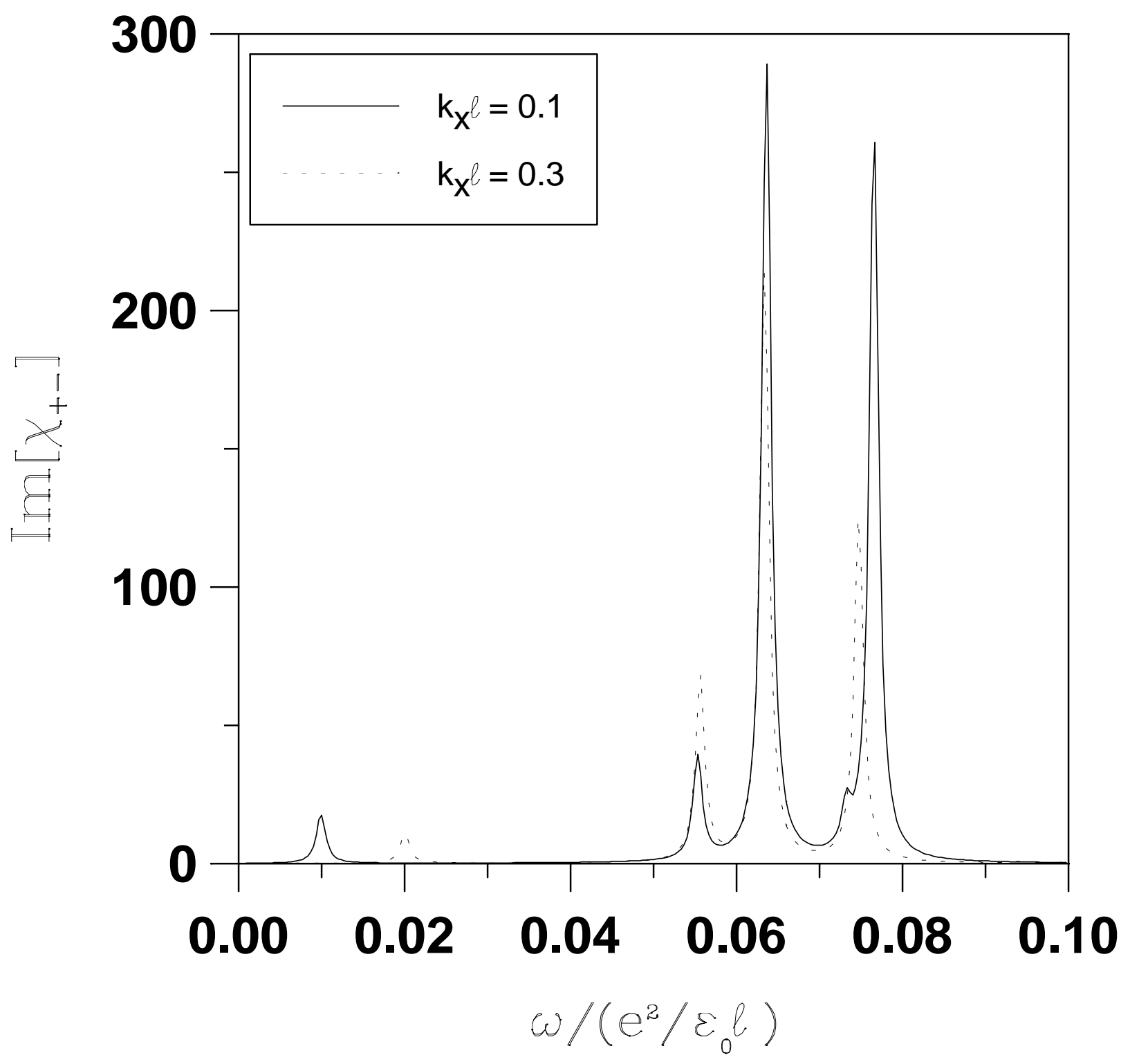

Fig. 8(b) R. Côté et al. 


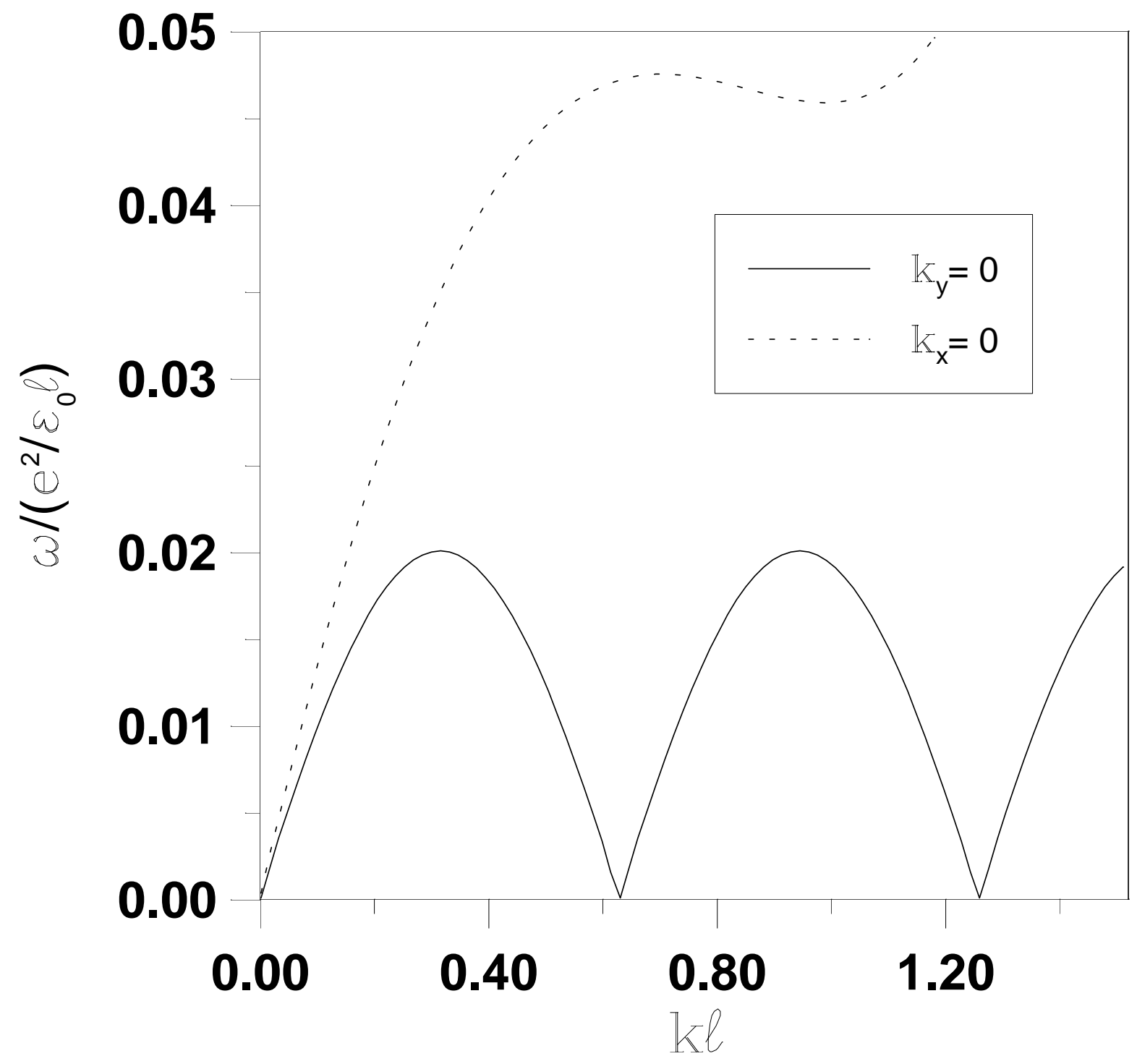

Fig. 9(a) R. Côté et al. 


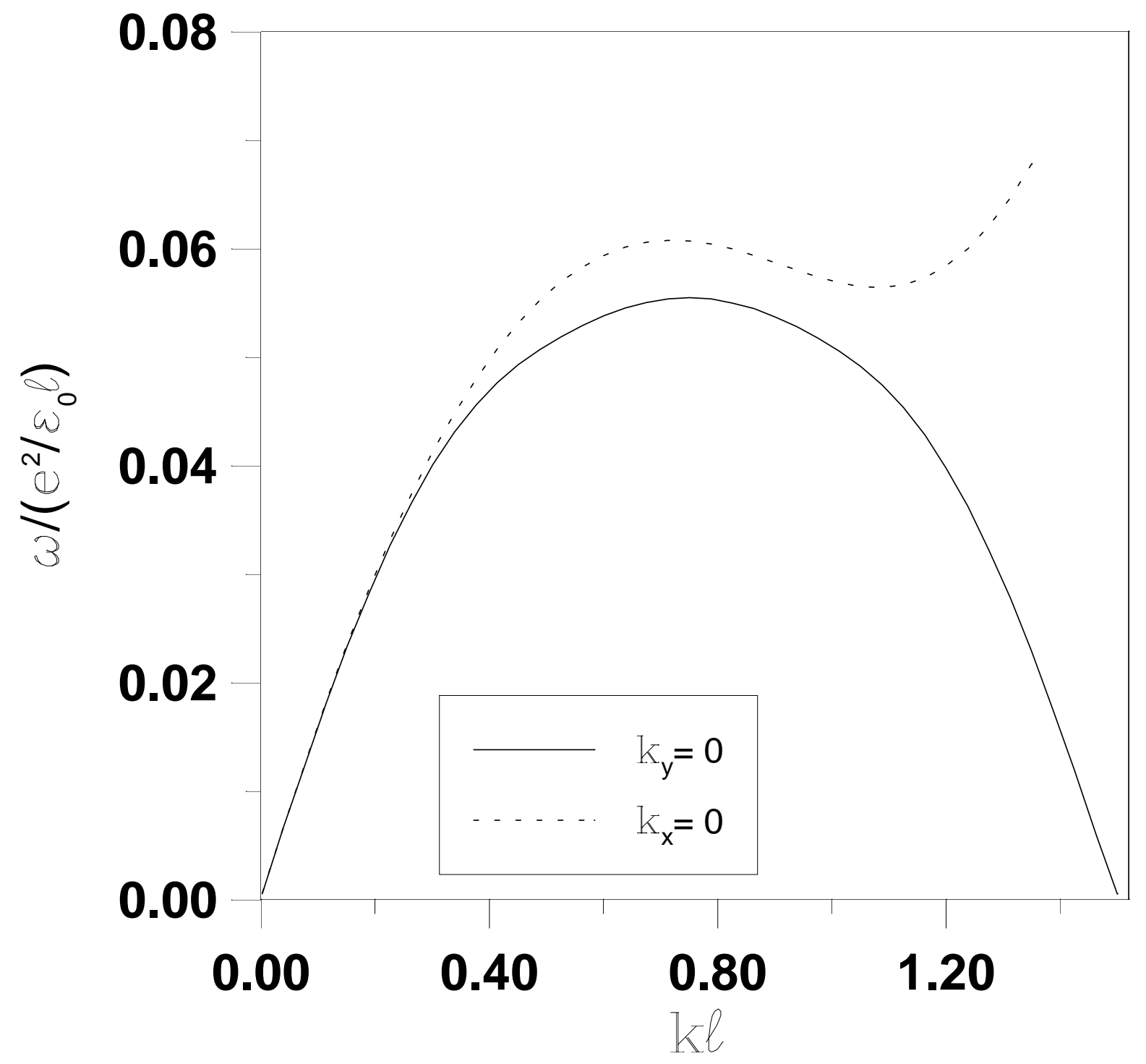

Fig. 9(b) R. Côté et al. 


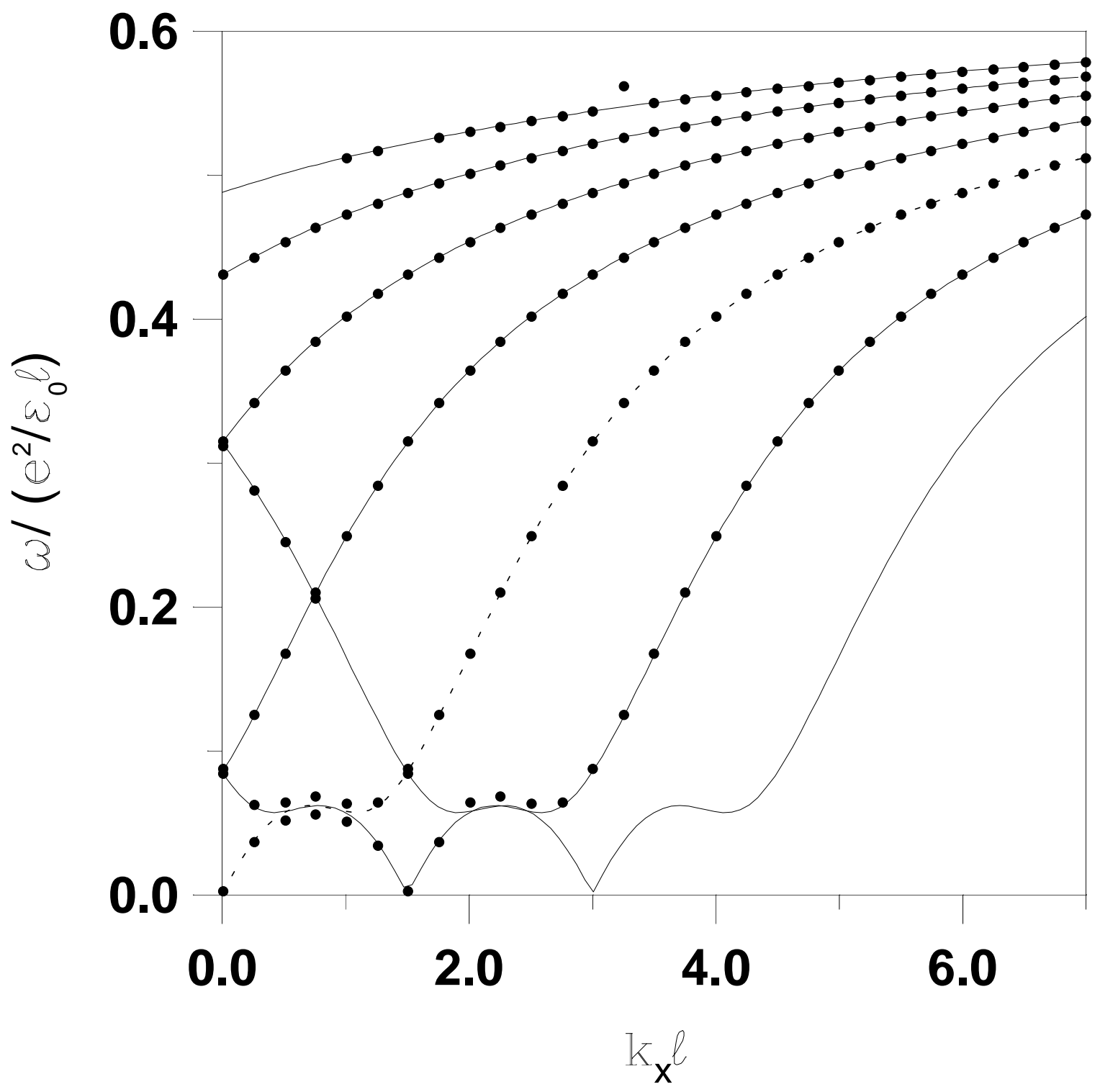

Fig. 10 R. Côté et al. 